Article

\title{
Numerical and Experimental Investigation on Radiated Noise Characteristics of the Multistage Centrifugal Pump
}

\author{
Qiaorui $\mathrm{Si}^{1}{ }^{1}$, Biaobiao Wang ${ }^{1}$, Jianping Yuan ${ }^{1}$, Kaile Huang ${ }^{1}$, Gang Lin $^{1}$ and \\ Chuan Wang 1,2,*(D) \\ 1 National Research Center of Pumps, Jiangsu University, Zhenjiang 212013, China; siqiaorui@ujs.edu.cn (Q.S.); \\ wbbujs@163.com (B.W.); yh@ujs.edu.cn (J.Y.); huangkaileujs@163.com (K.H.); lingangujs@163.com (G.L.) \\ 2 College of Hydraulic Science and Engineering, Yangzhou University, Yangzhou 225009, China \\ * Correspondence: wangchuan@ujs.edu.cn; Tel.: +86-150-508-54196
}

Received: 4 September 2019; Accepted: 25 October 2019; Published: 2 November 2019

\begin{abstract}
The radiated noise of the centrifugal pump acts as a disturbance in many applications. The radiated noise is closely related to the hydraulic design. The hydraulic parameters in the multistage pump are complex and the flow interaction among different stages is very strong, which in turn causes vibration and noise problems because of the strong hydraulic excitation. Hence, the mechanism of radiated noise and its relationship with hydraulics must be studied clearly. In order to find the regular pattern of the radiated noise at different operational conditions, a hybrid numerical method was proposed to obtain the flow-induced noise source based on Lighthill acoustic analogy theory, which divided the computational process into two parts: computational fluid dynamics (CFD) and computational acoustics (CA). The unsteady flow field was solved by detached eddy simulation using the commercial CFD code. The detailed flow information near the surface of the vane diffusers and the calculated flow-induced noise source was extracted as the hydraulic exciting force, both of which were used as acoustic sources for radiated noise simulation. The acoustic simulation employed the finite element method code to get the sound pressure level (SPL), frequency response, directivity, et al. results. The experiment was performed inside a semi-anechoic room with a closed type pump test rig. The pump performance and acoustic parameters of the multistage pump at different flow rates were gathered to verify the numerical methods. The computational and experimental results both reveal that the radiated noise exhibits a typical dipole characteristic behavior and its directivity varies with the flowrate. In addition, the sound pressure level (SPL) of the radiated noise fluctuates with the increment of the flow rate and the lowest SPL is generated at $0.8 Q_{d}$, which corresponds to the maximum efficiency working conditions. Furthermore, the experiment detects that the sound pressure level of the radiated noise in the multistage pump rises linearly with the increase of the rotational speed. Finally, an example of a low noise pump design is processed based on the obtained noise characteristics.
\end{abstract}

Keywords: multistage centrifugal pump; flow-induced noise; numerical calculation; acoustic analogy

\section{Introduction}

Multistage pumps with high pressure are widely used in water supply facilities [1-4]. The flow in the multistage pump is highly unsteady and greatly influenced by the interference between the different stages $[5,6]$. These give rise to the pressure pulsations, mechanical vibrations, and the radiated noise in various pump components [7-10]. Especially, the intensive pressure pulsations are an important source of hydrodynamic excitation force, which in turn produces fluid borne noise and structure-borne 
noise. Further, fluid-borne noise is a major contributor to radiated noise as well as leading to increased fatigue in the system components. Additionally, the radiated noise is felt as a disturbance in some applications and many conditions are subject to stringent requirements concerning the limitation of noise. Therefore, the investigation of the radiated noises from the centrifugal pump is significant to pump designers.

Computational fluid dynamics (CFD) has been used widely in many engineering fields with the rapid development of computer technology [11-16]. Previous experimental and computational study of the noise generation in the centrifugal pump assured that the noise source in the centrifugal pump is composed of the mechanical noise and flow-induced noise [17-19]. The radiation noise of the pump system can be significantly reduced by increasing the machining accuracy and using components such as low noise motors. However, the low-frequency noise induced by the fluid flow is very difficult to be eliminated. Recently, computational fluid dynamics were widely used to predict the flow-induced noise and analyze its corresponding sound source [20,21]. By employing the Lighthill's acoustic analogy theory, Howe [22] concluded that the dipoles source is the main source of the flow-induced noise in the centrifugal pump. In addition, Kato [23] proposed a one-way coupled simulation method that combines CFD and structure analysis. Ding [24] and Si [25] used the combined CFD/ computational acoustics (CA) method to simulate the hydraulic noise of centrifugal pumps. Keller [26] theoretically and experimentally investigated the effects of the pump-circuit acoustic coupling on the blade-passing frequency perturbation induced by fluid-dynamic interaction between the rotor and the stator, which provides some theoretical supports for the above numerical works. By employing the large eddy simulation (LES) and the Ffowcs Williams and Hawkings (FW-H ) acoustic method, Gao [27] concluded that the design operation generates the lowest total sound pressure level. Jiang [28] reported the fluid-induced noise by using the Fluid-solid coupling method and regarded it as a reliable way to predict the fluid-induced noise in the rotating machinery. Sergey [29] simulated the flow-induced noise based on the acoustic-vertex method, which divided the fluid mechanics equation into the sound item and the vortex item. Rual [30] studied the unsteady flow near the tongue and found that the size of the mesh, the time step, and the turbulence model had great influence on the accuracy of the sound simulation. Furthermore, a hybrid method that transforms the information of the unsteady flow into the source of the sound is widely used [31-33]. This method could improve simulation accuracy with lower computational resources [34]. To be specific, the detached eddy simulation (DES) model, which uses the RANS model to simulate the flow near the wall and employs the LES model to simulate the flow far away from the wall, could improve the computational efficiency and produce equal accurate unsteady results [35]. For the acoustic simulation, the finite element method (FEM) method could predict the acoustic distribution at the low frequency well and show more tolerance to the geometric irregularity.

The present paper focuses on the numerical and experimental investigation of the characteristics of the radiated noise of the multi-stage pump. The sound source was extracted from the unsteady computation based on the DES turbulence model, and the simulation code of acoustic finite element method inlaid in software Actran 14 was used to simulate the distribution of the radiated noise of the model pump. In addition, the change of the radiated noise with the variation of the flow rate and the rotation speed is also discussed.

\section{Method and Basic Theory}

\subsection{Theory of the Acoustic Simulation}

Acoustic analogies are derived from the Navier-Stokes equations, which governed both flow field and the corresponding acoustic filed. The Navier-Stokes equations expressed as Formula (1) and (2) are rearranged into various forms of the inhomogeneous acoustic wave equation.

$$
\frac{\partial \rho}{\partial t}+\frac{\partial}{\partial x_{i}}\left(\rho u_{i}\right)=0
$$




$$
\frac{\partial\left(\rho u_{i}\right)}{\partial t}+\frac{\partial\left(\rho u_{i} u_{j}\right)}{\partial x_{j}}=-\frac{\partial p}{\partial x_{i}}+\frac{\partial e_{i j}}{\partial x_{j}}
$$

where $e_{i j}$ is the viscous stress tensor, and the last Lighthill function is expressed as:

$$
\frac{\partial^{2}\left(\rho-\rho_{0}\right)}{\partial t^{2}}-c_{0}^{2} \frac{\partial^{2}}{\partial x_{i} \partial x_{j}}\left(\rho-\rho_{0}\right)=\frac{\partial^{2} T_{i j}}{\partial x_{i} \partial x_{j}}
$$

where $T_{i j}$ is the stress tensor, and $T_{i j}=\rho u_{i} u_{j}-e_{i j}+\delta_{i j}\left[\left(p-p_{0}\right)-c_{0}^{2}\left(\rho-\rho_{0}\right)\right] ; \delta_{i j}$ is the Kronecker function, $\rho$ is the fluid density, $\rho_{0}$ is the undisturbed density, $c_{0}$ is the sound velocity, $t$ is the time, $x$ is the space coordinates, while the $i, j$ represent the direction of the coordinate axis.

Furthermore, by employing several mathematical manipulations, the above function could be transformed as:

$$
\int_{\Omega}\left(\frac{\partial^{2}}{\partial t^{2}}\left(\rho-\rho_{0}\right) \delta \rho+c_{0}^{2} \frac{\partial}{\partial x_{i}}\left(\rho-\rho_{0}\right) \frac{\partial(\delta \rho)}{\partial x_{i}}\right) d x=-\int_{\Omega} \frac{\partial T_{i j}}{\partial x_{j}} \frac{\partial(\delta \rho)}{\partial x_{i}} d x+\int_{\Gamma} \frac{\partial \sum_{i j}}{\partial x_{j}} n_{i} \delta \rho d \Gamma(x)
$$

The first term of the right is the volume source, and the second term is the surface source. In the acoustic simulation, the solution of the Navier-Stokes equation firstly assumes the water as incompressible to calculate the flow-induced acoustic source. Then, the compressibility of the water is to be considered to solve the acoustic wave propagation.

In addition, the description of the radiated noise is related to time and space. Mathematically, this relationship could be expressed as an acoustic wave equation. The process of the sound radiation meets the basic physical law, which is expressed as:

$$
\begin{gathered}
\left(\rho_{0}+\rho^{\prime}\right)\left(\frac{\partial}{\partial t}+v \nabla\right) v=-\nabla\left(p_{0}+p^{\prime}\right) \\
\frac{\partial\left(\rho_{0}+\rho^{\prime}\right)}{\partial t}=\left(\rho_{0}+\rho^{\prime}\right) q^{\prime}-\left(\rho_{0}+\rho^{\prime}\right) \nabla v \\
p^{\prime}=\frac{\gamma p_{0}}{\rho_{0}} \rho^{\prime}+\frac{\gamma(\gamma-1)}{2 \rho_{0}^{2}}\left(\rho^{\prime}\right)^{2}
\end{gathered}
$$

where, $\rho_{0}, v_{0}$ and $p_{0}$ respectively represent the static density, static velocity, and static sound pressure. And $\rho^{\prime}, v^{\prime}, p^{\prime}$, and $q^{\prime}$, respectively represent the increment of the density, velocity, sound pressure, and the mass. With some mathematical treatment, the acoustic wave equation is expressed as:

$$
\nabla^{2} p^{\prime}=\frac{1}{c^{2}} \frac{\partial^{2} p^{\prime}}{\partial t^{2}}-\rho_{0} \frac{\partial q^{\prime}}{\partial t}
$$

where $\nabla^{2}=\frac{\partial^{2}}{\partial x^{2}}+\frac{\partial^{2}}{\partial y^{2}}+\frac{\partial^{2}}{\partial z^{2}}$ and $c=\sqrt{\gamma \frac{p_{0}}{\rho_{0}}}$, which is the sound velocity in the fluid.

The vibration at any time could be decomposed as several simple harmonic vibrations expressed as:

$$
\begin{aligned}
& p^{\prime}=p(x, y, z) e^{j w t} \\
& q^{\prime}=q(x, y, z) e^{j w t}
\end{aligned}
$$

A combination of the above three equations could deduct the acoustic wave equation expressed as;

$$
\nabla^{2} p(x, y, z)-k^{2} p(x, y, z)=-j \rho_{0} \omega q(x, y, z)
$$

where $k=\frac{2 \pi f}{c}$ and this value represents the number of the waves, $\omega$ is the angular velocity. 


\subsection{DES Method}

The DES method is a modification of a RANS model in which the model switches to a subgrid-scale formulation in regions fine enough for LES calculations, which calculates the sound source information and simultaneously cuts down the cost of the computation. It is initially formulated for the Spalart-Allmaras model, which is expressed as:

$$
\frac{D \tilde{v}}{D t}=c_{b 1} \tilde{S} \tilde{v}+\frac{1}{\sigma}\left[\nabla \cdot\left((v+\tilde{v}) \cdot \nabla \tilde{v}+c_{b 2}(\nabla \tilde{v})\right]^{2}-c_{w 1} f_{w}\left[\frac{\tilde{v}}{d_{w}}\right]^{2}\right.
$$

where the variables that represent turbulent motion are the quantities directly solved by the S-A equation. The relationship with the motion viscosity coefficient is defined as:

$$
v_{t}=\frac{\mu_{t}}{\rho}=\tilde{v} f_{v 1}
$$

where $f_{v 1}$ is a dimensionless function defined as:

$$
f_{v 1}=\frac{\chi^{3}}{\chi^{3}+c_{v 1}^{3}}, \chi=\frac{\tilde{v}}{v}
$$

where $v$ is an expression of the molecular viscosity generation term expressed as:

$$
\tilde{S}=f_{v 3} S+\frac{\tilde{v}}{\kappa^{2} d_{w}^{2}} f_{v 2}
$$

where $S$ is the absolute value of vorticity, and $f_{v 2}$ and $f_{v 3}$ express dimensionless functions, which is respectively expressed as:

$$
f_{v 2}=\left(1+\frac{\chi}{c_{v 2}}\right)^{-3}, f_{v 3}=\frac{\left(1+\chi f_{v 1}\right)\left(1-f_{v 2}\right)}{\chi}
$$

$f_{w}$ is expressed as:

$$
f_{w}=g\left(\frac{1+c_{w 3}^{6}}{g^{6}+c_{w 3}^{6}}\right)^{\frac{1}{6}}, g=r+c_{w 2}\left(r^{6}-r\right), r=\frac{\tilde{v}}{\tilde{S} \kappa^{2} d_{w}^{2}}
$$

Constant value is evaluated as:

$$
\begin{aligned}
& c_{b 1}=0.1355, c_{b 2}=0.622, \sigma=2 / 3, \kappa=0.41, \\
& c_{w 1}=\frac{c_{b 1}}{\kappa^{2}}+\frac{1+c_{b 2}}{\sigma}, c_{w 1}=0.3, c_{w 3}=2, c_{v 1}=71
\end{aligned}
$$

DES method is based on the S-A model which replaces the feature-length $d_{w}$ as:

$$
\tilde{d}=\min \left(d_{w}, C_{D E S} \Delta\right)
$$

where $\Delta=\max (\Delta x, \Delta y, \Delta z)$ represents the maximum grid distance, $C_{D E S}=0.6$.

The default value of the software is adopted in the article. The size of the characteristic length is related to the scale of the grid, and the DES method is an S-A model of the LES simulation.

\subsection{Simulation Procedure}

Considering that the flow field inside the multistage pump is complex and the acoustic simulation domain is irregular, the finite element method is used in this acoustic simulation which could avoid the inaccuracy during the transformation of the sound source. Firstly, the computational flow domain and corresponding structured grids were prepared to process the steady simulation of the pump flow field based on the experimental inlet and outlet boundary conditions. Simultaneously, the test rig 
was built to use the obtained pump performance data such as the pump head to verify or modify the numerical calculation model. The shear stress transport (SST) turbulence model embedded in ANSYS CFX 14.1 software (ANSYS, Inc., Commonwealth of Pennsylvania, USA) was repeatedly used at this step. Afterwards, the correct fluid calculation domain and the appropriate grid were obtained. Secondly, the unsteady simulation of the pump flow field was obtained using the DES method and the detailed flow information, such as flow velocity, pressure, density etc, was extracted and transformed as the sound source in the acoustic simulation. Meanwhile, the acoustic calculation domains were built and the acoustic meshes were generated containing an interface setting. Finally, the radiated noise calculation was completed by the acoustic finite element method using Actran12.0 software (Free Field Technologies MSC Software Company, Mont-Saint-Guibert, Belgium). The acoustic characteristics of the model pump were analyzed after experimental verification. The flow chart of the simulation work is shown in Figure 1.

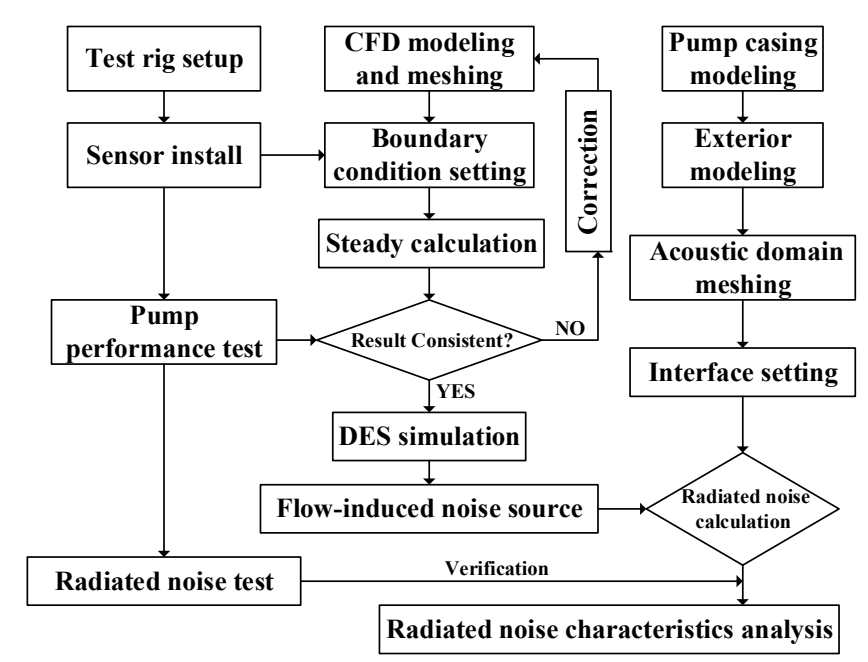

Figure 1. The flow chart of the radiated noise calculation of the multi-stage centrifugal pump.

\section{Numerical Method}

\subsection{Study Object}

The study object is focused on a self-priming multistage pump type, which is widely used to feed water to the high floors of a building. The field measurement revealed that this type of multistage pump generated a higher sound pressure level than the technical requirements. The basic analysis of this problem showed the noise is caused by the hydraulic design. The geometry features of the example multistage pump with five stages in this study is shown in Figure 2a. Its inflow is axial and its outflow is along the radial direction. Each stage of this pump is composed of one impeller and one vane diffuser. The impeller constitutes seven backswept blades, and the vane diffuser is two-dimensional with 12 guiding vanes and 12 returning vanes. The design impeller rotation speed of the pump is $2800 \mathrm{r} / \mathrm{min}$ and the design flow rate of the pump is $8 \mathrm{~m}^{3} / \mathrm{h}$. The single-stage head of the pump is $10 \mathrm{~m}$ and the total head is a superposition of the stages. The detailed hydraulic parameters of each stage are presented in Table 1. In order to simplify the model, this study only takes the three-stages hydraulic part of the pump and makes a prototype as shown in Figure $2 b$ for the simulation and experimental test. 


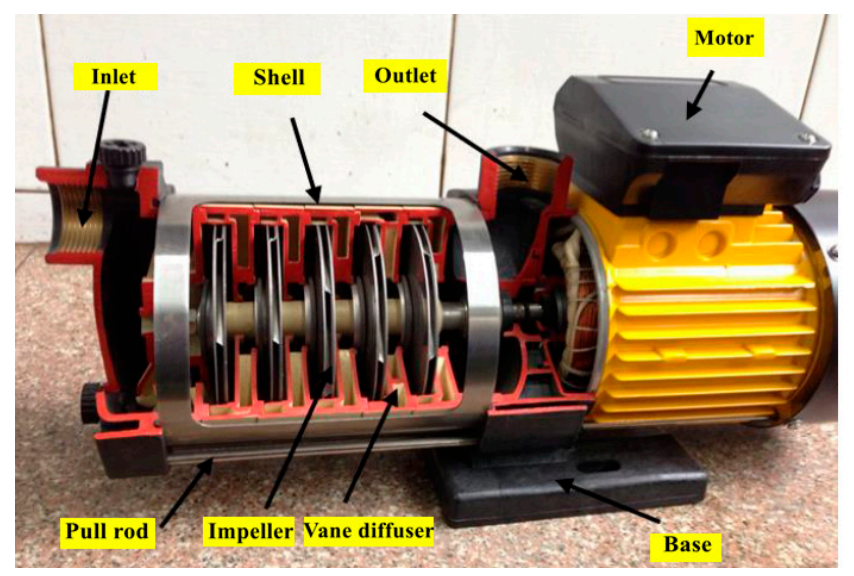

(a) The cutaway view of the multi-stage centrifugal pump prototype.

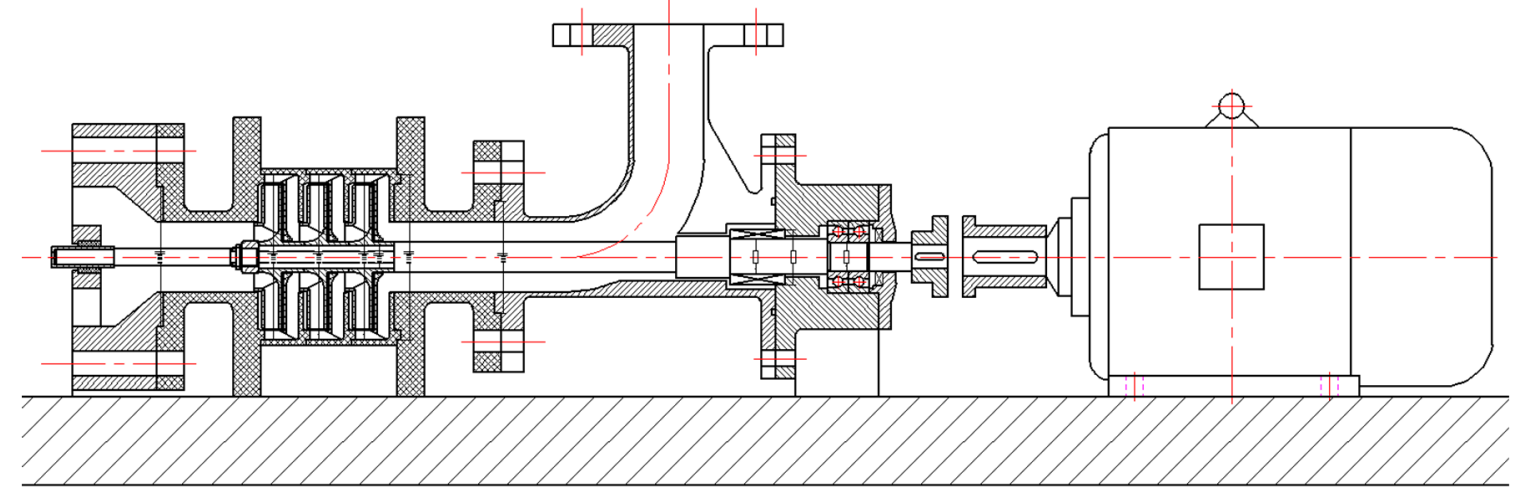

(b) Test prototype of the simplified model pump.

Figure 2. Pump model.

Table 1. Main characteristics of the model pump.

\begin{tabular}{cccc}
\hline Geometry & Values & Geometry & Values \\
\hline Impeller inlet diameter, $D_{j}$ & $45 / \mathrm{mm}$ & Blade outlet angle, $\beta_{2}$ & $37 /^{\circ}$ \\
Impeller outlet diameter, $D_{2}$ & $103 / \mathrm{mm}$ & Impeller blades number, $Z_{a}$ & 7 \\
Impeller outlet width, $b_{2}$ & $10 / \mathrm{mm}$ & Diffuser vane number, $Z_{d}$ & 12 \\
Diffuser inlet diameter, $D_{3}$ & $104.5 / \mathrm{mm}$ & Specific speed, $n_{\mathrm{s}}$ & 86 \\
\hline
\end{tabular}

\subsection{Fluid Field Simulation}

\subsubsection{Computational Domain}

In general, the multistage pump is composed of the first stage, the last stage, and several middle stages. To save the computational resources, the number of the middle stages was reduced to one stage and the first and last stages were still kept, as presented in Figure 3. Such simplification is appropriate to reflect the physical problems of the study object because the flow in the middle stages is similar except the initial pressure. Each computational stage includes one impeller, one vane diffuser, and the gap between the impeller and vane diffuser. Apart from the three stages, the computational stage is composed of the inlet section, the wear-ring section, pump cavity, impeller, vane diffuser, and the outlet section, which are consistent with the flow path of the experimental model pump. Both the inlet and outlet suction are extended appropriately to ensure the numerical stability, simultaneously to comply with the experimental boundary conditions. 


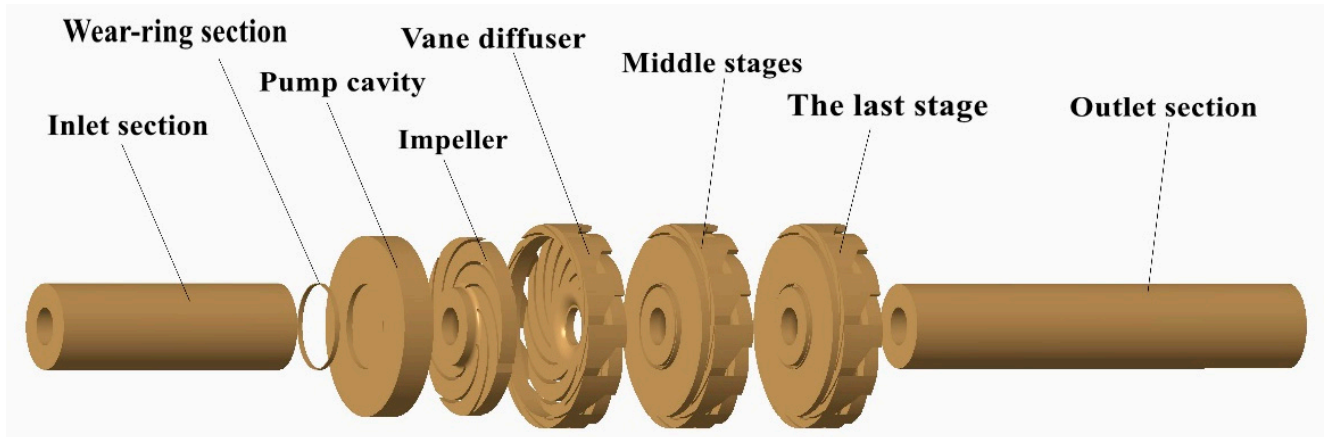

Figure 3. Domains of the flow field calculation.

\subsubsection{Mesh Generation and Boundary Conditions}

The structured mesh is applied for the computational domains by using ANSYS ICEM version 14.1 (ANSYS, Inc., Commonwealth of Pennsylvania, USA). As shown in Figure 4, a special refinement is applied around the blades and vane diffusers to improve the accuracy of the simulation. In addition, six groups of computational grids have been chosen to analyze the influence of the mesh size on the prediction of the hydraulic performance of the model pump. Figure 5 presents the analysis of mesh independence verification, the total element number is chosen as 6,680,830 by considering the simulation accuracy and efficiency. In addition, boundary conditions concern a bar at the inlet and the mass flow rate at the outlet of the computational domain. All the other walls are treated as non-slip boundaries. The maximum nondimensional wall distance, $y+<10$, was obtained in the complete flow field, which could satisfy the requirement of all turbulence modeling methods used in this paper. The pre-converged steady flow field (based on the SST turbulence model) obtained is accepted as the initial condition followed by the unsteady simulation (based on the DES model). The time step for the unsteady simulation is set as $1.7857 \times 10^{-4} \mathrm{~s}$.

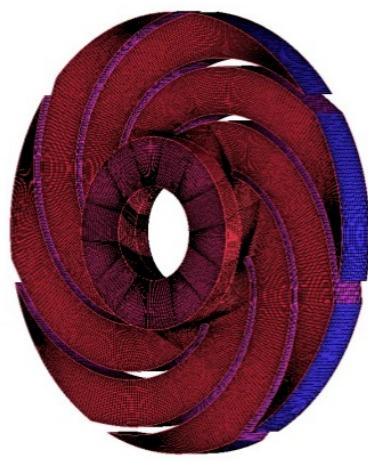

(a) impeller

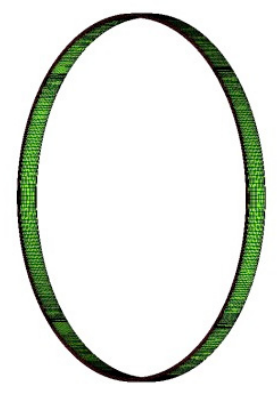

(d) wear-ring

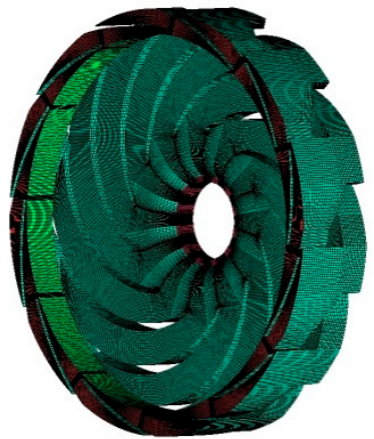

(b) vane diffuser

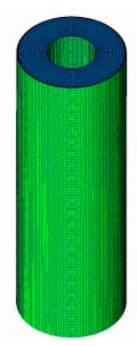

(e) inlet section

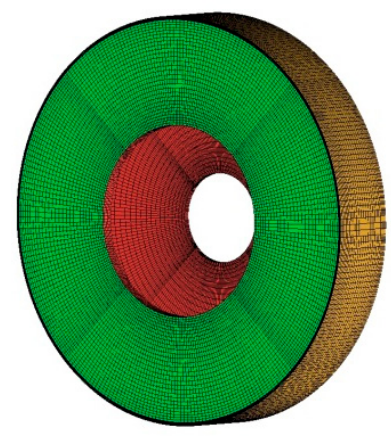

(c) gap of the pump

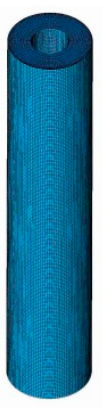

(f) outlet section

Figure 4. Mesh of the multi-stage centrifugal pump. 


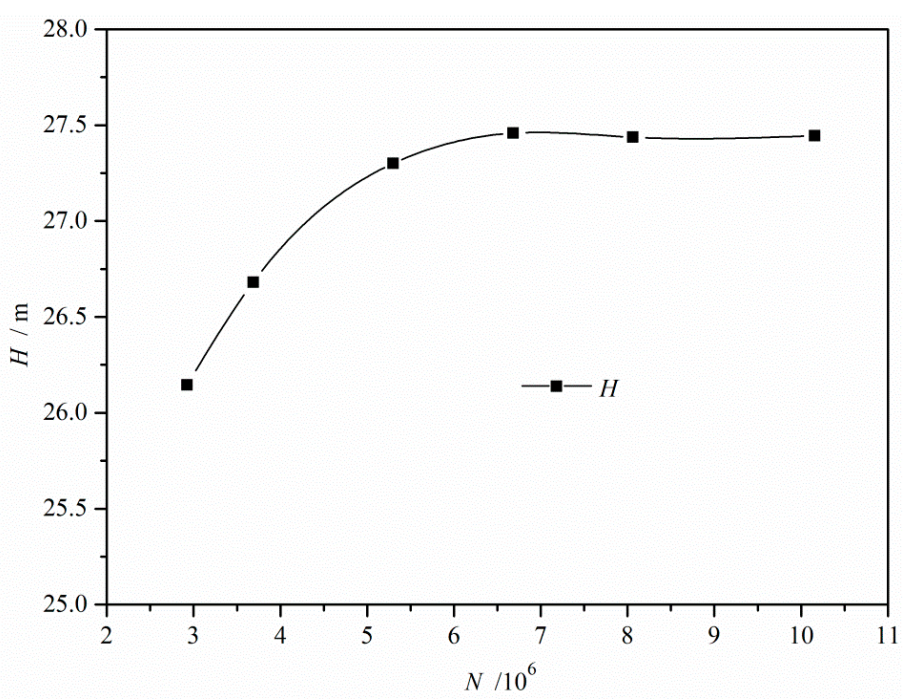

Figure 5. Analysis of mesh independence.

\subsubsection{Flow Field Results}

The global performance of the model pump is shown in Figure 6. The experimental and simulated data of the total head agree well with nearby design flow rates. The calculated head is slightly different from the measured value at the part-load and overload condition. The value of calculated efficiency is lower than the measured one and the differences become bigger with the increment of the flow rate. While the error of the head between the experiment and the simulation is lower than $4.5 \%$ and the error of the efficiency is lower than 3.8\%, the established calculation model and the selected number of grids could fully support the next acoustic calculations. By the way, the highest efficiency working condition of the model pump appears in the $0.8 Q_{d}$. This might be related to subsequent acoustic characterization.

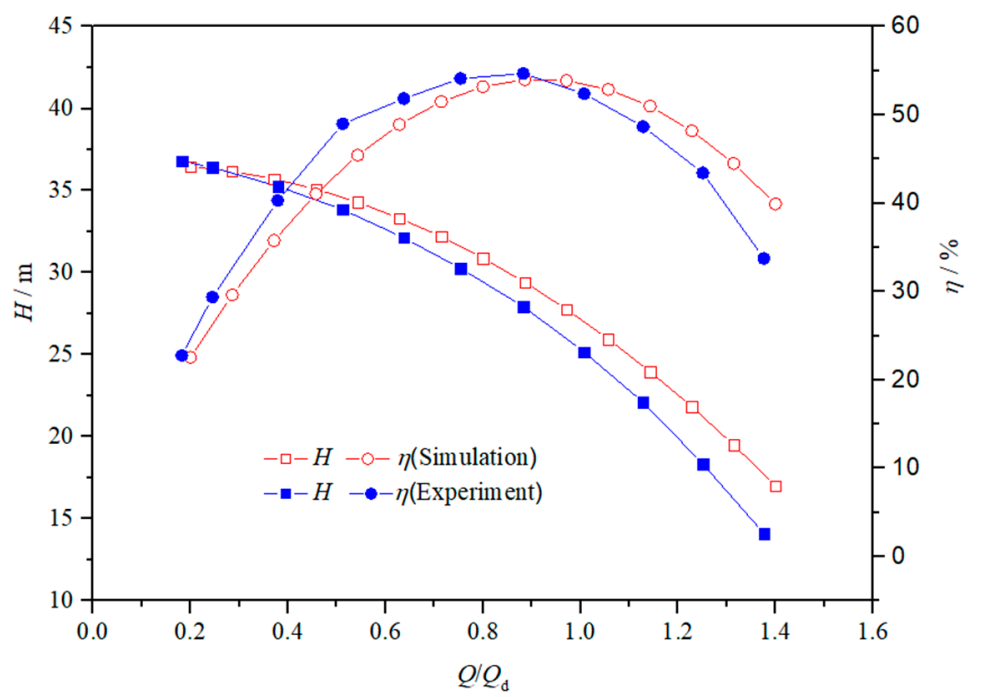

Figure 6. External characteristics of numerical calculation and experiment.

Figure 7a-d describes the velocity distribution on the mid-span of the impeller and the diffuser. At the $0.6 Q_{d}$ condition, the velocity distribution in the impeller is not uniform and the separation is detected at the inlet of the diffuser. With the increase of the flow rate, the flow field becomes better distributed while the diffusers are impugned by the high-velocity flow. The wake flow induced by the impingement can generate higher pressure pulsations. As pointed out by Gülish [36], the wake flow and the separation in the centrifugal pump gives rise to the pressure pulsations and the subsequent radiated noise. 

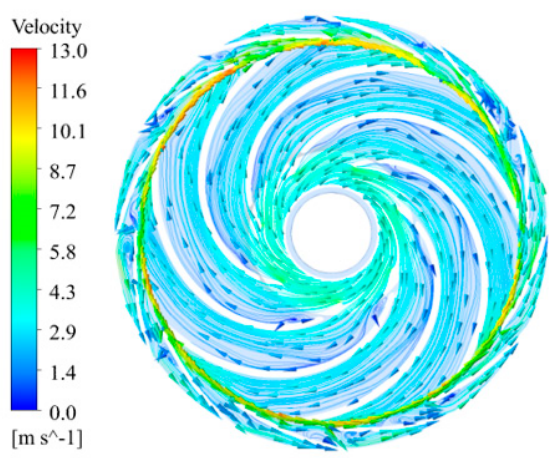

(a) 0.6 $Q_{\mathrm{d}}$ at impellers and positive vanes
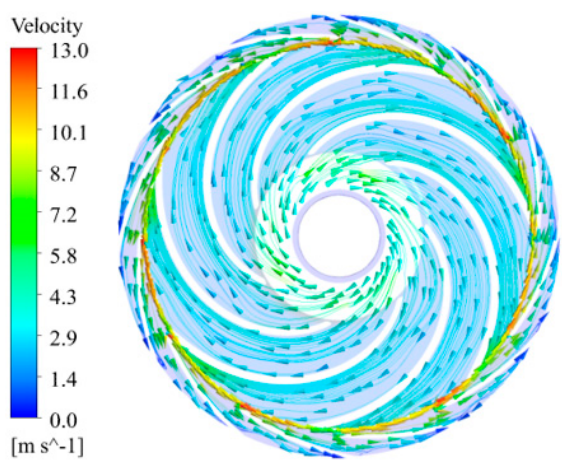

(c) $1.0 Q_{\mathrm{d}}$ at impellers and positive vanes
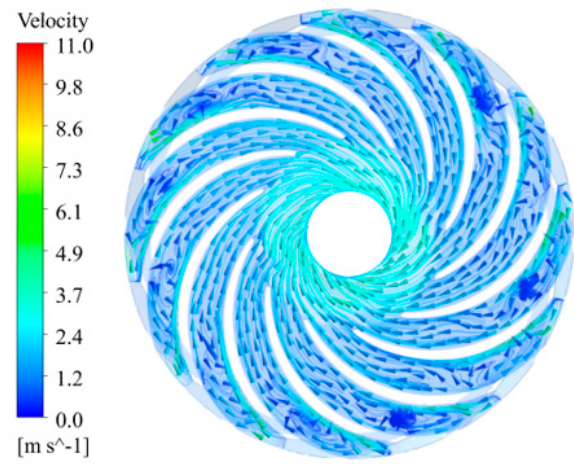

(e) $0.6 Q_{d}$ at reverse vanes

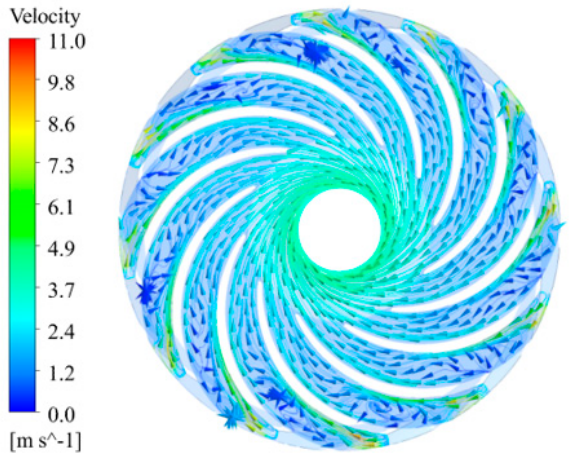

(g) $1.0 Q_{d}$ at reverse vanes

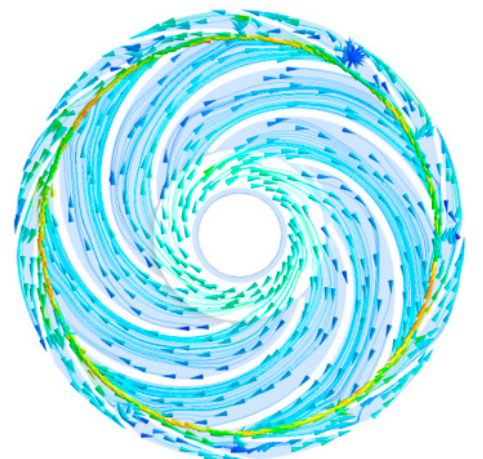

(b) $0.8 Q_{d}$ at impellers and positive vanes

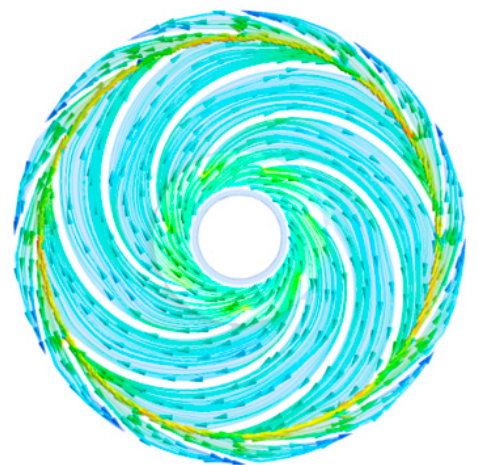

(d) $1.2 Q_{d}$ at impellers and positive vanes

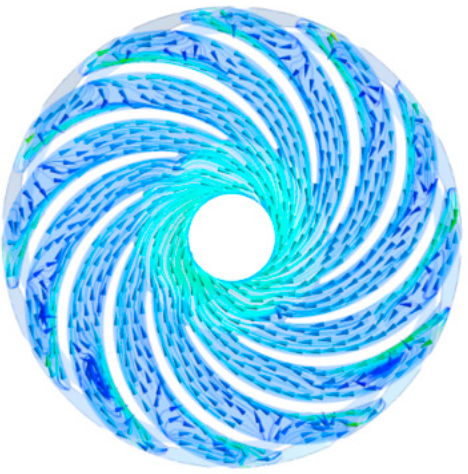

(f) $0.8 Q_{d}$ at reverse vanes

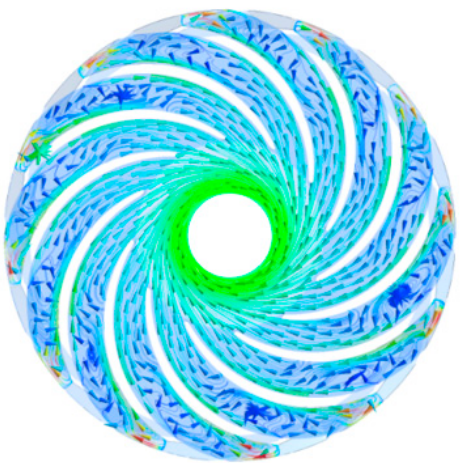

(h) $1.2 Q_{d}$ at reverse vanes

Figure 7. Velocity distribution in the mid-scan of the pump's first stage at different flowrates. 
Considering the influence of the upstream flow, the flow in the returning vane diffuser passages is more complex, as shown in Figure 7e-h. At the part-load condition, several separations block several passages. Additionally, the flow is greatly deaccelerated in the diffuser passages at the overload condition and the separation flow is detected near the suction side of the diffuser. When the flow reaches $0.8 Q_{\mathrm{d}}$, the number of separations is the fewest. This might indicate that minor noise would produce at this flow condition.

In order to understand the pressure pulsation characteristics of the flow in the model pump at various stages of the multistage centrifugal pump, 12 monitoring points at every stage, totaling 36 points, are set on the cross-section of the impeller, the positive vane, and the reverse guide vane. As shown in Figure 8, the first letter in each monitoring point name is: $F$ means the first level, $S$ means the second level, and $T$ means the third level. Moreover, the second letter in each monitoring point name is: $Y$ means the impeller, $D$ means the positive vane, and $F$ means the reverse guide vane. The dimensionless pressure pulsation coefficient $C_{p}{ }^{*}$ as shown in Formula (20) is used for further data reduction.

$$
C_{P}^{*}=\frac{(p-\bar{p})}{0.5 \rho v_{2}^{2}}
$$

where $p$ is the static pressure of the monitoring point, $\bar{p}$ is the average value of the static pressure, $\rho$ is the fluid density, and $v_{2}$ is the circumferential velocity at the impeller outlet. Afterward, standard deviation $\mathrm{C}_{\mathrm{PS}}$ was used to characterize the pressure pulsation intensity of each monitoring point, and fast Fourier transform processing was used to obtain the frequency spectrum of the flow pressure fluctuation.

As shown in Figure 8b, the pressure pulsation intensity of the monitoring points in the multi-stage centrifugal pump shows a periodic change, and it always became larger around the positive guide vane flow channel, indicating that the flow between the stages is similar and the biggest hydraulic exciting force appears at the positive guide vane. The maximum value of the pressure pulsation intensity appears on monitoring points near the throat of the positive guide vane, indicating that the rotor-stator interaction between impeller and positive guide vane makes the greatest contribution to the pressure pulsation intensity. Other flow conditions show the same results. For more detail, the maximum pressure pulsation intensity appears in the first-stage positive guide vane flow channel, and the secondary and final guide vanes make less difference. Then, frequency analysis of the pressure fluctuation at different flow rates is processed on the monitoring points of the first positive guide vane, as shown in Figure 8c-f. Since the sampling time step of the numerical calculation is $1.7857 \times 10^{-4} \mathrm{~s}$, the sampling frequency is $5600 \mathrm{~Hz}$. According to the Nyquist sampling theorem, the maximum frequency that can be analyzed is $2800 \mathrm{~Hz}$.
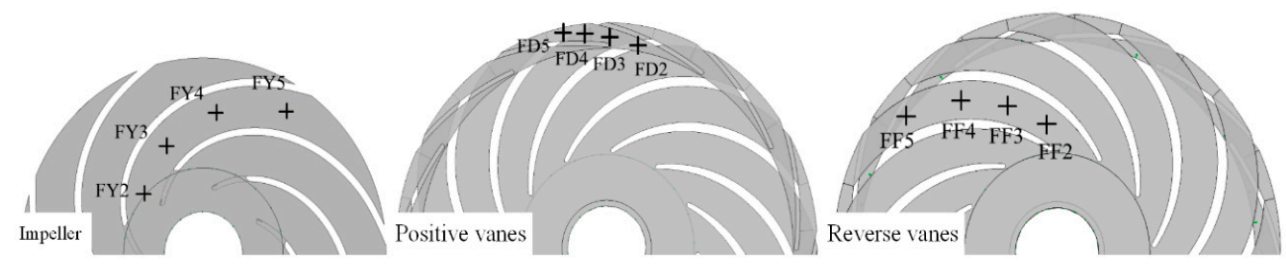

(a) monitoring points at the first stage of the model pump

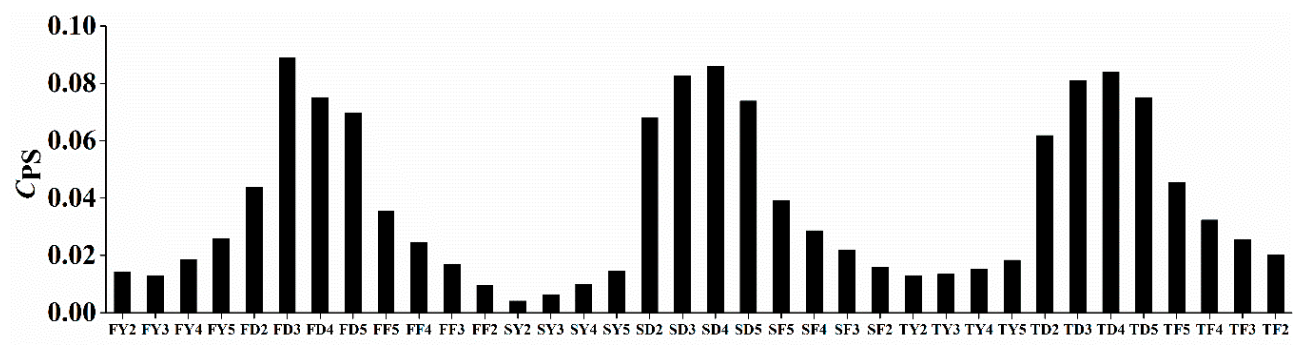

(b) amplitude distribution of pressure fluctuation at $1.0 Q_{\mathrm{d}}$

Figure 8. Cont. 


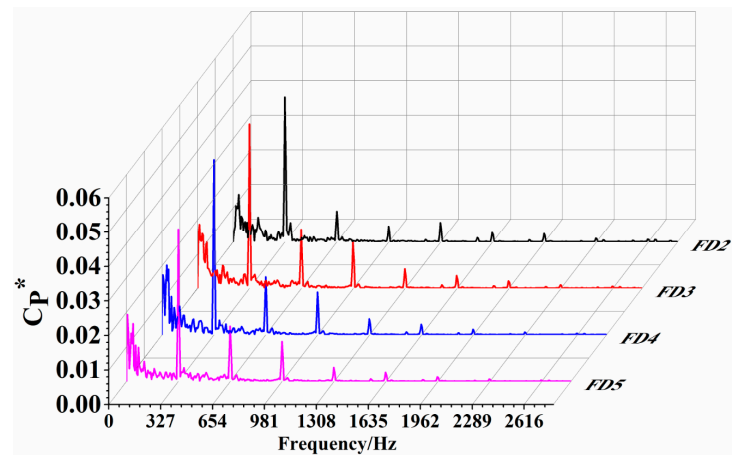

(c) spectrum of pressure fluctuation at $0.6 Q_{d}$

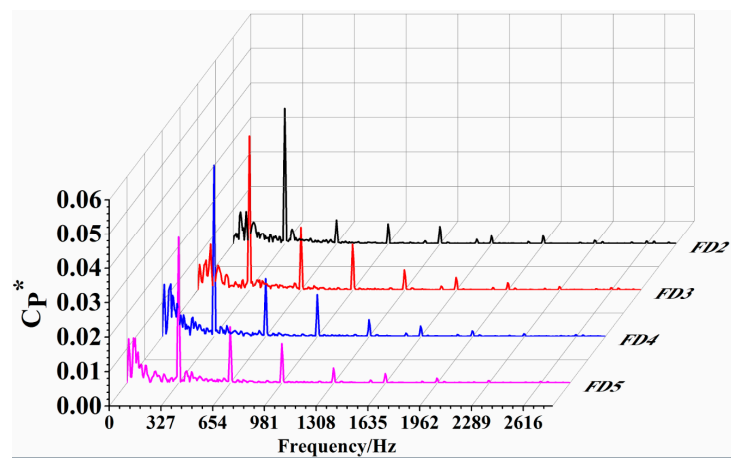

(d) spectrum of pressure fluctuation at $0.8 Q_{d}$

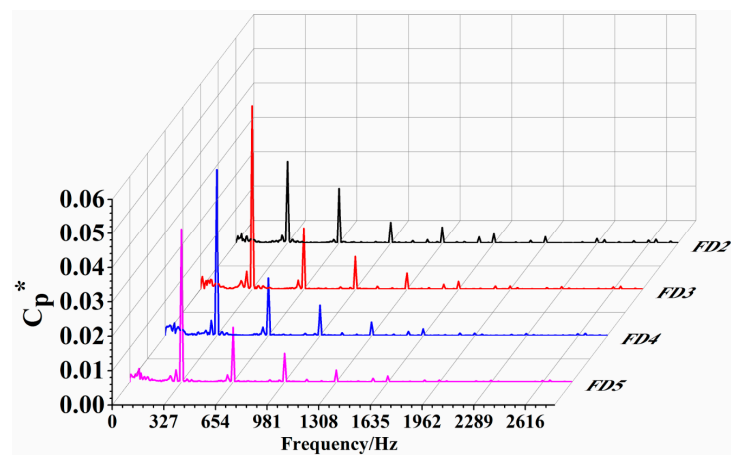

(e) spectrum of pressure fluctuation at $1.0 Q_{\mathrm{d}}$

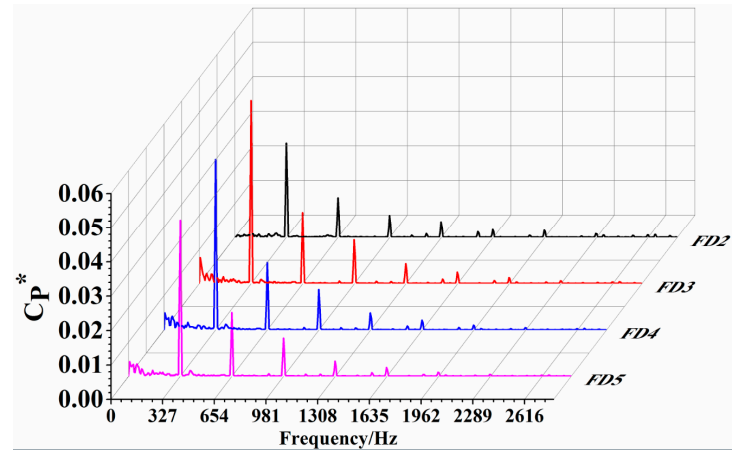

(f) spectrum of pressure fluctuation at $1.2 Q_{\mathrm{d}}$

Figure 8. Pressure fluctuation of monitoring points. 
The results of the frequency spectrum show that the pressure pulsation is mainly concentrated in the low-frequency region within $1000 \mathrm{~Hz}$, and the main frequency is the blade passing frequency $(327 \mathrm{~Hz})$ and its multiple. The monitoring point with a large peak is also located near the throat of the positive guide vane at all flow rates, which corresponds to the pressure pulsation intensity results. Under the small flow conditions such as $0.6 Q_{d}$ and $0.8 Q_{d}$, there are many low-frequency pulsations smaller than the blade passing frequency for all monitoring points. It means the periodic pressure pulsation caused by the rotor-stator interaction between impeller and positive guide vane is the major source of the pump unstable operation. The $0.8 Q_{d}$ has the lowest amplitude of the frequency spectrum, which corresponds to the phenomenon that this flowrate present the highest efficiency.

\subsection{Radiated Noise Analysis}

\subsubsection{Computational Domain}

The computational domain for the acoustic simulation includes the structural domain and air-borne domain, as shown in Figure 9. The inner surface of the vane diffuser of the structural domain is loaded with information of the unsteady flow to get the sound source in the subsequent simulation, and the definition of the air-borne domain is to get the distribution of the radiated noise in the subsequent simulation.

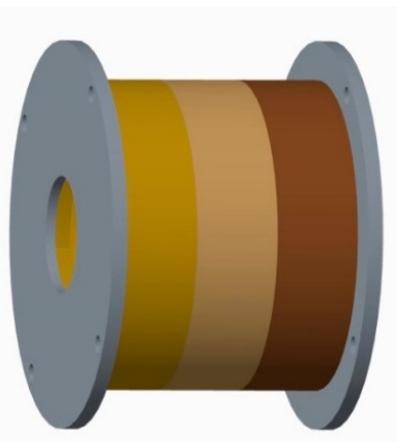

(a) structural domain

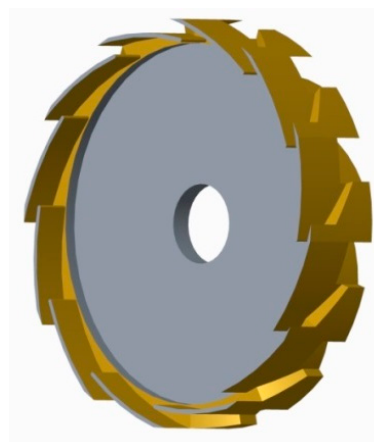

(c) the structure of the vane diffuser

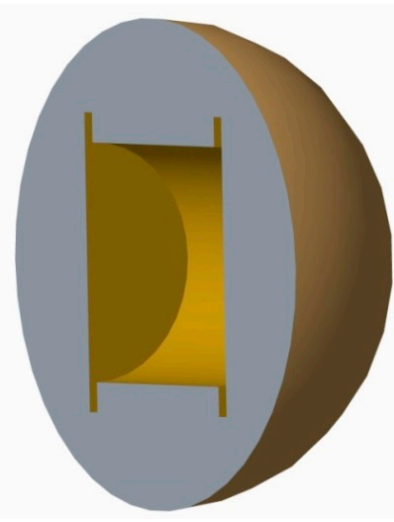

(b) air-borne domain

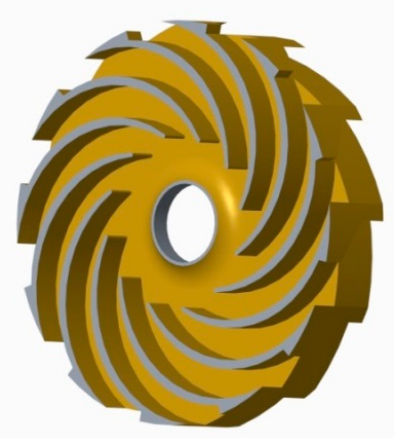

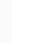

$$
\text { (1) }
$$

Figure 9. Calculation domains of radiated noise simulation.

\subsubsection{Mesh Generation and Boundary Condition}

Unstructured mesh, which has better adaptability to the geometry, is applied in the acoustic simulation. To guarantee the precision of the acoustic computation, the maximum mesh size should meet the Formula (21). In this study, the maximum mesh size is $0.008 \mathrm{~m}$. And the mesh for acoustic simulation is shown in Figure 10. 


$$
L_{\max }<\frac{c}{6 f_{\max }}
$$

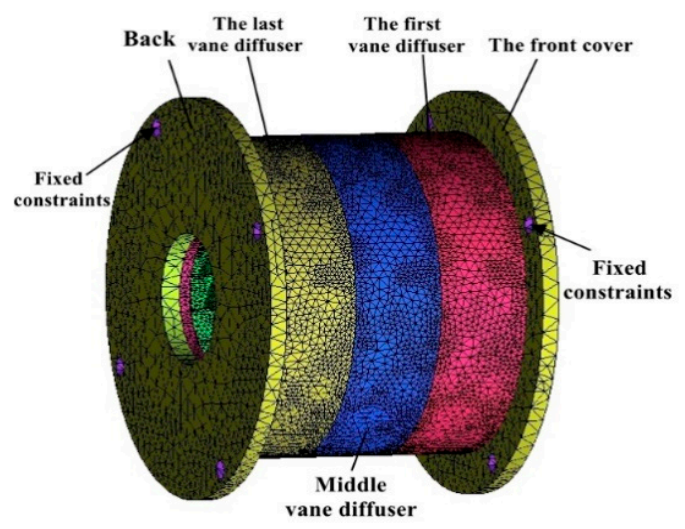

(a) mesh for the structural domain

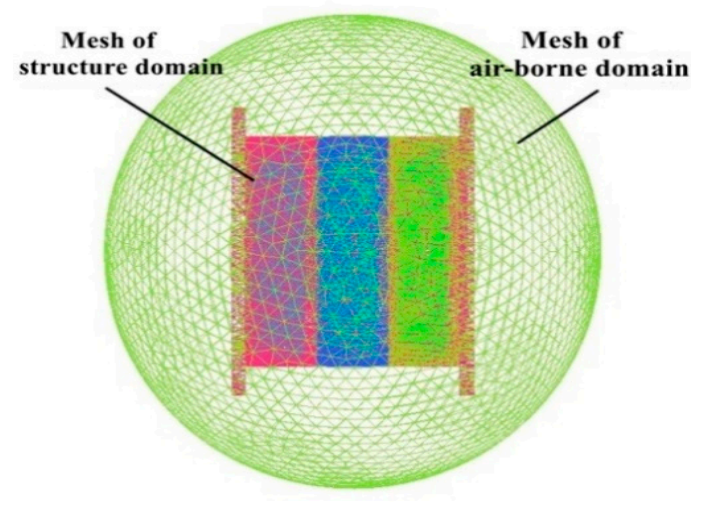

(b) acoustic simulation mesh

Figure 10. The sound field calculation mesh of multistage centrifugal pump.

The detailed information of the unsteady flow is extracted as the sound source near the surface of the vane diffusers. In addition, the material properties of the structure domain are shown in Table 2. Considering the total time of the unsteady CFD simulation and the time step, the frequency range of the acoustic simulation is set from $0 \mathrm{~Hz}$ to $2800 \mathrm{~Hz}$, and the resolution is set as $5.3 \mathrm{~Hz}$. The data transmission of the interface between the structural domain and the air-borne domain is finished with the integral interpolation method. In order to analyze the properties of the radiated noise, 60 monitor points are mounted equally in the mid-span surface of the second impeller and distances between the diffuser surfaces of these points are $1 \mathrm{~m}$, as shown in Figure 11.

Table 2. Material properties of the structure domain.

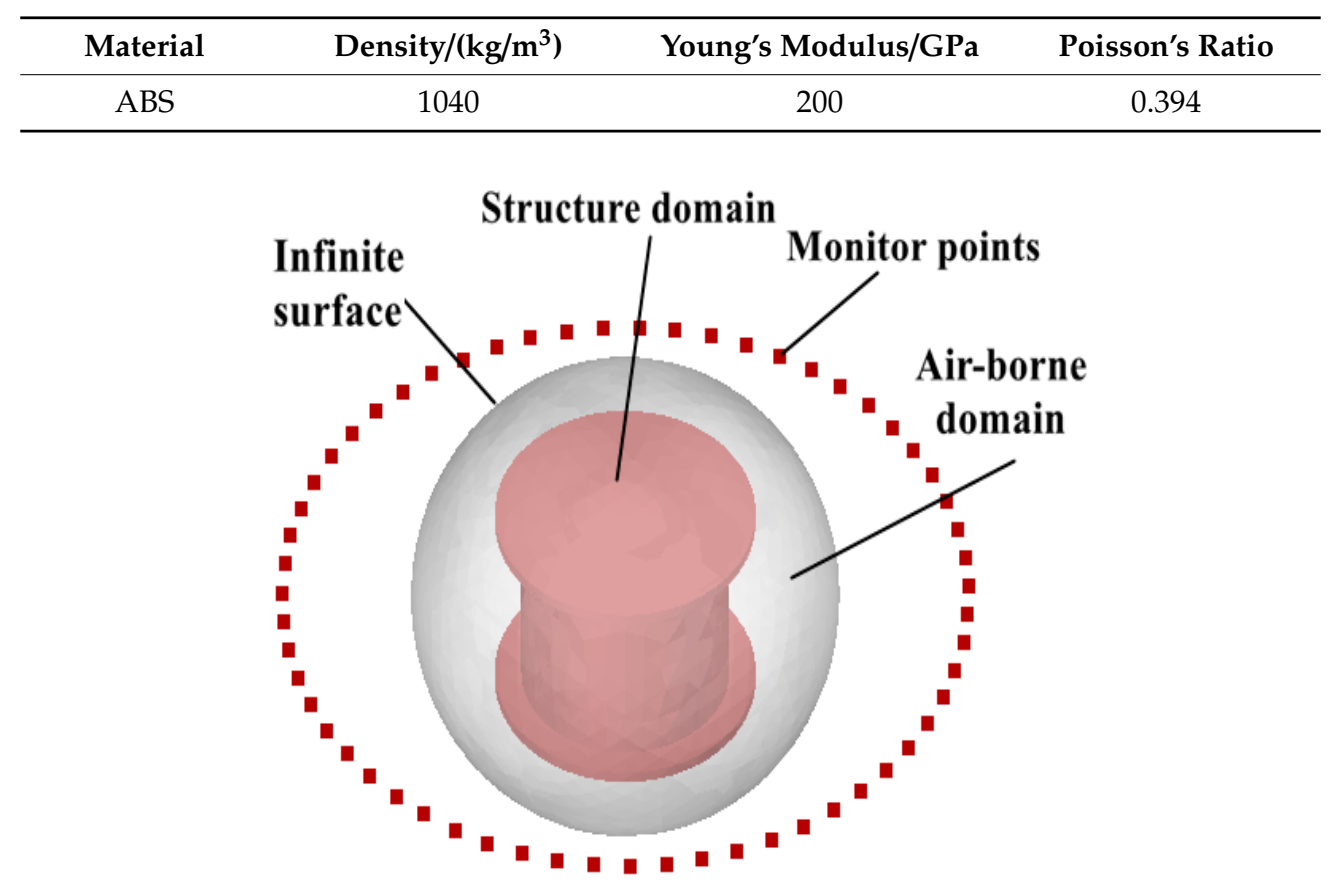

Figure 11. The pre-setting of radiated noise calculation. 


\subsubsection{Acoustic Field Results}

Figure 12 shows the predicted sound pressure spectra at different flow rates. As can be seen, the dominant frequency of the multi-stage pump is the blade passing frequency $(327 \mathrm{~Hz})$. Furthermore, the sound pressure level (SPL) of the radiated noise at the low frequency increases with the increase of the flow rate. In contrast, the SPL above $2700 \mathrm{~Hz}$ has the opposite trend. Additionally, the SPL at the blade passing frequency decreases slightly before $0.8 Q_{\mathrm{d}}$, and increases afterward. In addition, there is a slight increase in the SPL around $1500 \mathrm{~Hz}$, which is assumed to be linked with the vibration mode of the pump system.

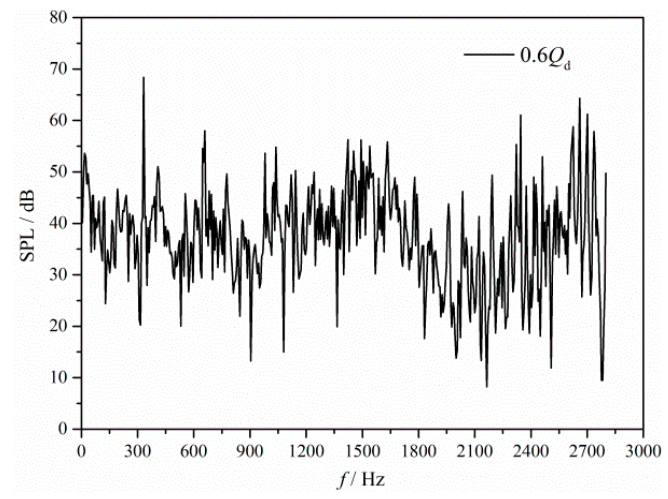

(a) $0.6 Q_{\mathrm{d}}$

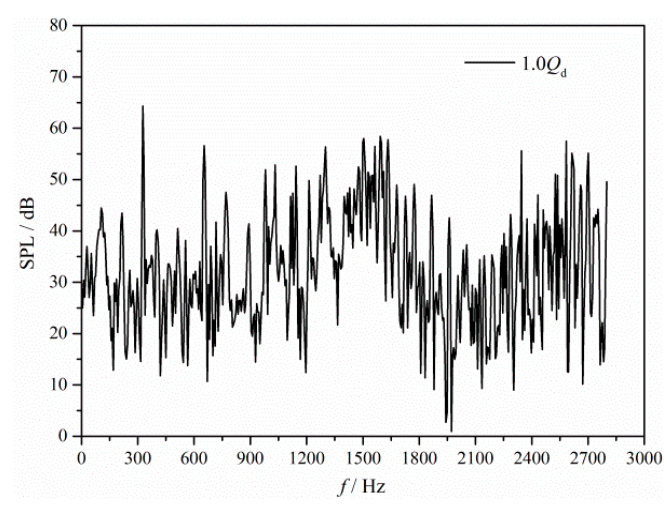

(c) $1.0 Q_{\mathrm{d}}$

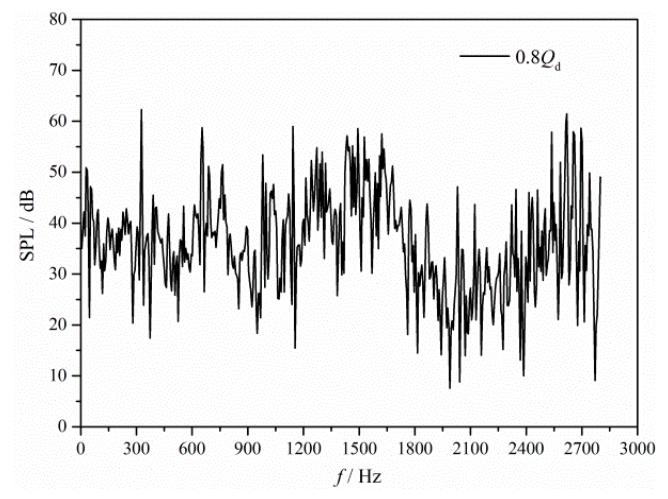

(b) $0.8 Q_{\mathrm{d}}$

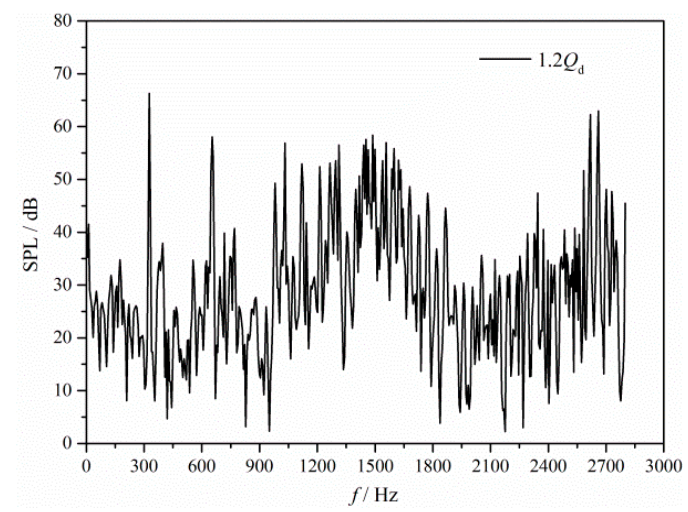

(d) $1.2 Q_{\mathrm{d}}$

Figure 12. Frequency response curves of radiated noise.

Figure 13 shows the sound pressure level contour at blade passing frequency. The sound pressure level reaches the highest at the $0.6 Q_{d}$, while the sound pressure level reaches the highest near the vane diffuser of the first stage. This is due to the fact that the intensity of the pressure fluctuation at the first stage is highest and the structural strength is relatively lower around the first stage.

The profiles of the SPL of the directivity field shown in Figure 14 demonstrate the dipole characteristic behavior. It is found that two SPL valleys appear around at $\theta=120^{\circ}$ and $300^{\circ}$ and fluctuate slightly with the change of the flowrate. This phenomenon illustrates that the rotor/stator interaction is the main source of the fluid-induced noise. 

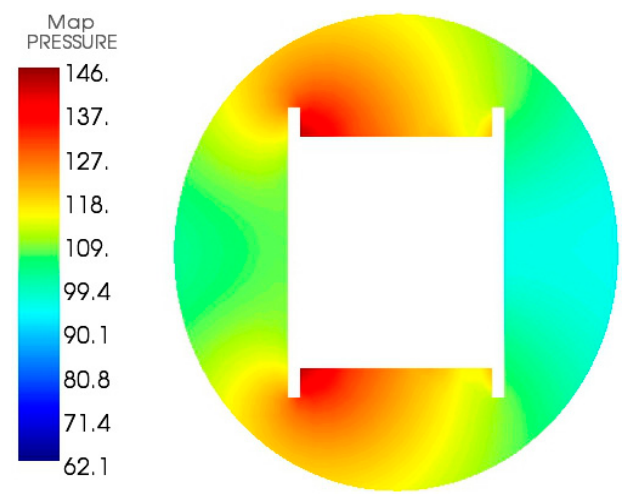

(a) $0.6 Q_{d}$

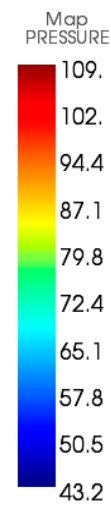

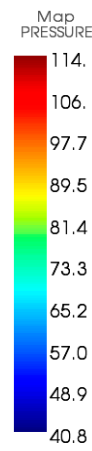

(b) $0.8 Q_{d}$

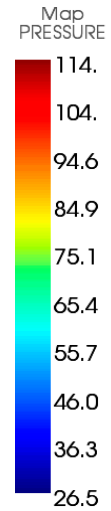

(d) $1.2 Q_{\mathrm{d}}$

(c) $1.0 Q_{\mathrm{d}}$

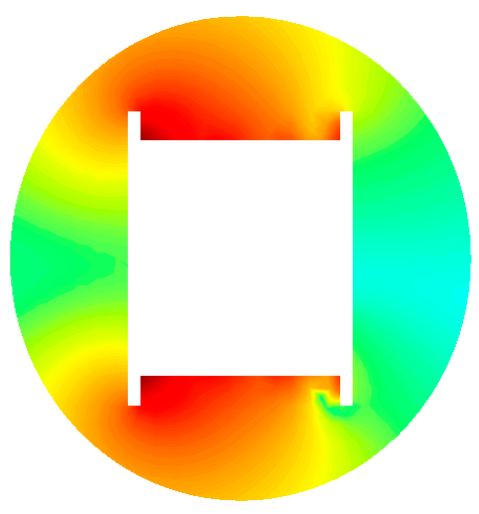

Figure 13. The sound pressure level contour at blade passing frequency.

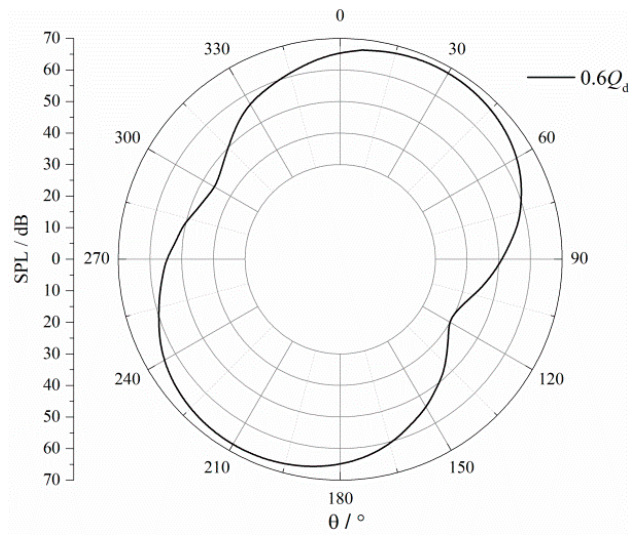

(a) $0.6 Q_{d}$

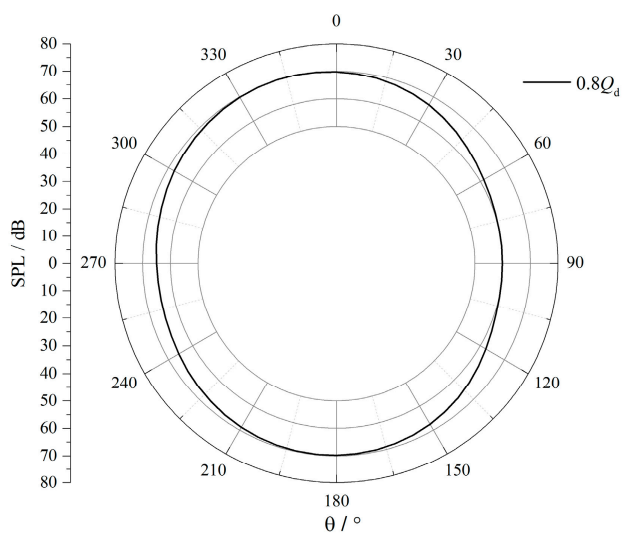

(b) $0.8 Q_{d}$

Figure 14. Cont. 


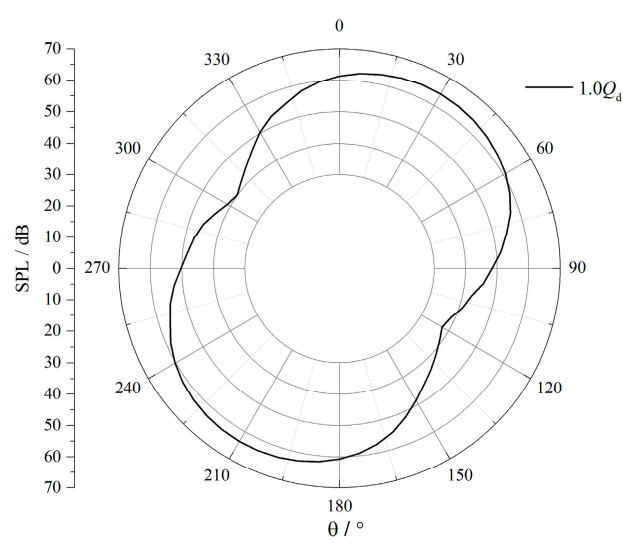

(c) $1.0 Q_{d}$

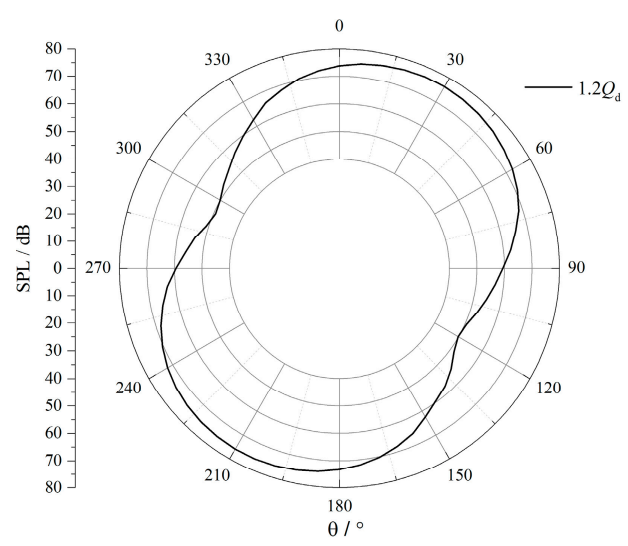

(d) $1.2 Q_{\mathrm{d}}$

Figure 14. The directivity of the radiated noise field at blade passing frequency.

\section{Experimental Verification}

The experimental research was done in a closed-loop test rig beside the semi-anechoic room of the National Research Center of Pumps, China. The test rig shown in Figure 15 for pump performance measurement meets the Grade 2 accuracy based on the ISO 9906.2012 standard. The accuracy of the flow rate measurements is $\pm 2.5 \%$, the head is $\pm 3 \%$, the torque is $\pm 2.5 \%$, and the rotation is $\pm 1 \%$. In addition, to verify the validity of the acoustic simulation results, the radiated noise of the multi-stage pump is measured in the semi-anechoic room built with $15 \mathrm{~dB}$ background noise and $50 \mathrm{~Hz}$ cut-off frequency. As shown in the above figure, the five walls of this semi-anechoic room are equipped with an anechoic wedge that could form a semi-free acoustic field. In order to improve the accuracy of the measurement, the multi-stage pump is installed on a damping base and the pipelines are supported with the damping disc. In addition, an anechoic tank is arranged between the water tanks and the multi-stage pump to reduce the flow-induced noise inside the pipelines. All the data collection equipment is put in the monitoring room to minimize the influence of the accuracy of the measurement.

The data collection system is composed of the module to measure the static characteristic and the other module to capture the radiated noise of the model pump. The magnetic inductive flowmeter performs the measurements of the volume flow, pressure sensors capture the static pressure at the inlet and outlet of the multi-stage pump and monitor the torque value of the model pump. The noise is measured with the PCB 14043 type microphone (PCB Piezotronics Inc., New York, USA) and processed based on the LMS test Lab platform. The accuracy of the SPL of the radiated noise is $\pm 1 \mathrm{~dB}$. The acquisition sampling frequency is set to $6000 \mathrm{~Hz}$, and the resolution frequency is set to $0.5 \mathrm{~Hz}$. Every test used $120 \mathrm{~s}$ for the signal acquisition and treated frequency resolution by the Hanning window in general.

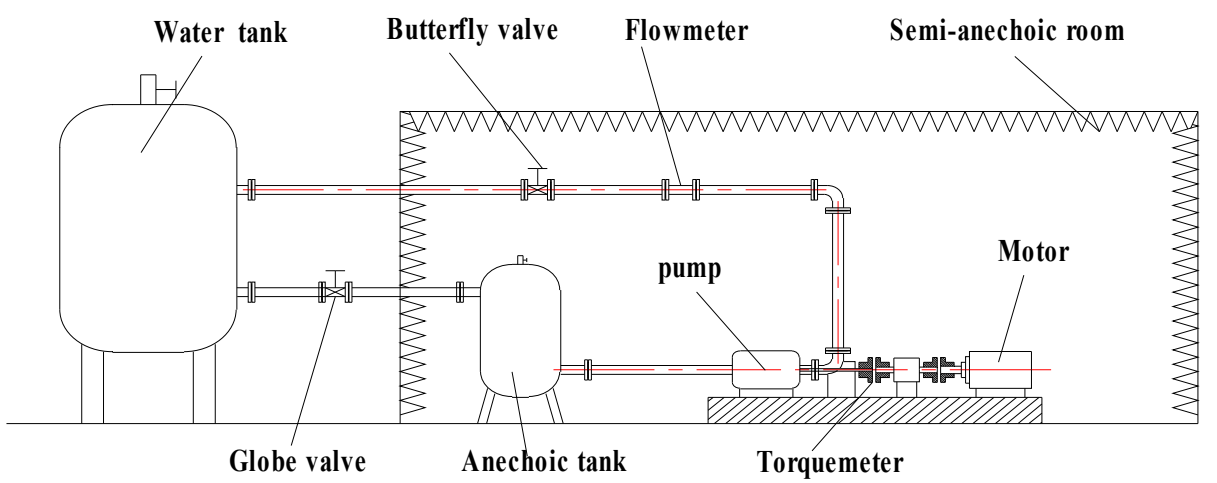

(a) test rig

Figure 15. Cont. 


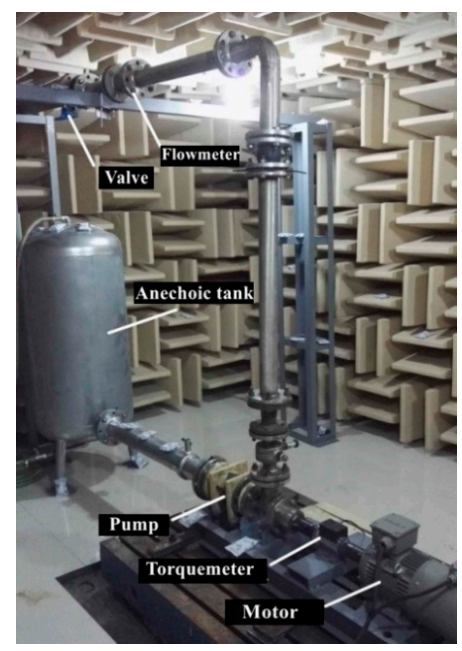

(b) model pump and the layout of the pipelines

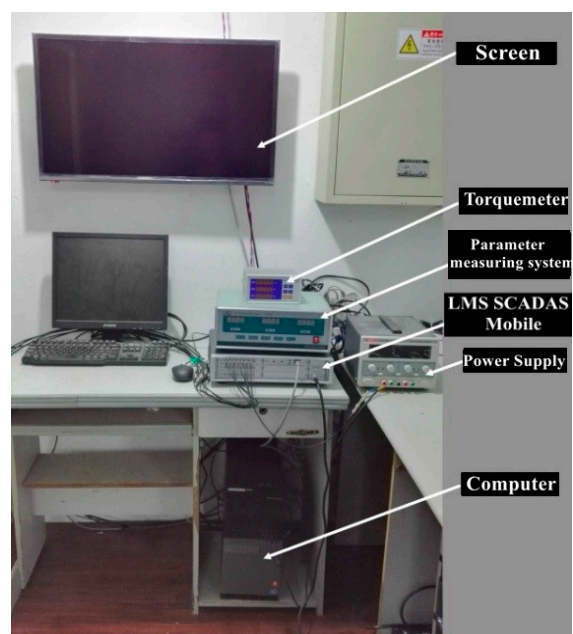

(c) monitoring room

Figure 15. Test of the multi-stage centrifugal pump.

\subsection{Radiated Noise at the Different Flow Rate}

To investigate the relationship between the radiated noise and flow rate, the flow rate is regulated by the butterfly valve to detect the radiated noise between $3 \mathrm{~m}^{3} / \mathrm{h}-11.6 \mathrm{~m}^{3} / \mathrm{h}\left(0.375 Q_{\mathrm{d}}-1.45 Q_{\mathrm{d}}\right)$. Five positions set for the microphones followed the Chinese standard GB/T 29529-2013 [37], as shown in Figure 16a, consistent with the position of the monitoring point set in the numerical simulation. Figure $16 \mathrm{~b}$ shows the setup with enclosures. Figure 17 shows the radiated noise of the model pump at different flowrates concerning the above two conditions and the comparison with the numerical simulation results. In order to analyze the variation of the total sound pressure level of the radiated noise of the multi-stage centrifugal pump, the average total sound pressure level $\bar{L}_{P}$ is expressed as follows. Where $N$ represents the number of sound monitoring points and $L_{\mathrm{pi}}$ is the total sound pressure level of each sound monitoring point.

$$
\bar{L}_{P}=10 \cdot \lg \left[\frac{1}{N} \sum_{i=1}^{N} 10^{0.1 L_{P_{i}}}\right]
$$

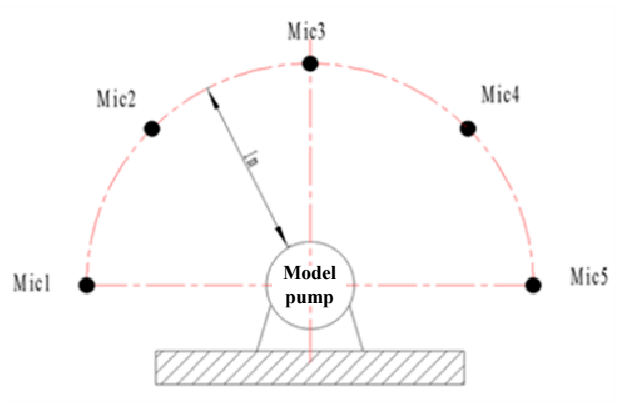

(a) location of the microphone

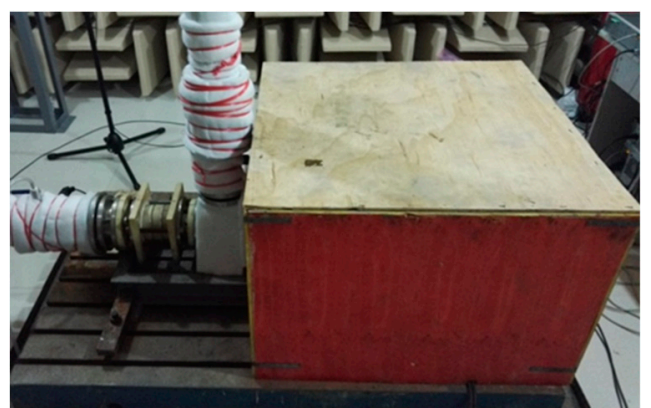

(b) with acoustic enclosure

Figure 16. Experiment method. 


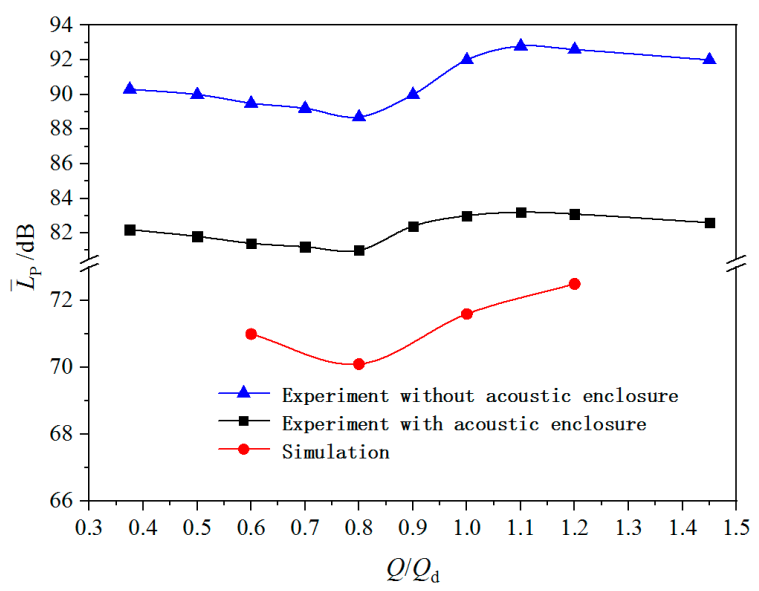

Figure 17. The radiated noise of the multi-stage centrifugal pump at different flowrates.

As seen in Figure 17, the radiated noise of the model pump at all flow rates with acoustic enclosure is $8 \mathrm{~dB}$ lower than the case without the enclosure. This fact proves that the enclosure is necessary for the experiment. On the other hand, the simulated total sound pressure level is consistent with the experiment that the total sound pressure level fluctuates with the increment of the flow rate and the minimal value emerges around $0.8 Q_{d}$ where the efficiency is the highest. As pointed out by Gülish [24], the sound pressure level of the induced noise is in the inverse relationship with the efficiency. The differences between the simulation and experiment are within the order of $10 \mathrm{~dB}$ because the background noise inside the anechoic chamber still provides some disturbance. Although we used sound elimination materials on the pipe system, the motor still emits a strong radiated noise even when we used an enclosure.

\subsection{Radiated Noise at the Different Rotational Speed}

The rotational speed of the centrifugal pump has the direct influence of the pressure distribution in the model pump and meantime influence on the radiated noise of the model pump. A frequency converter allows changing the rotational speed of the pump. As seen in Figure 18, the sound pressure level increases almost linearly with the increase of the rotational speed. Processed by the fitting instruments, their relation meets the following formula:

$$
y=0.00545 x+66.7
$$

In this formula, $y$ represents the sound pressure level, $x$ represents rotational speed, and the linear dependence between the fitted curve and the measured data is 0.97894 .

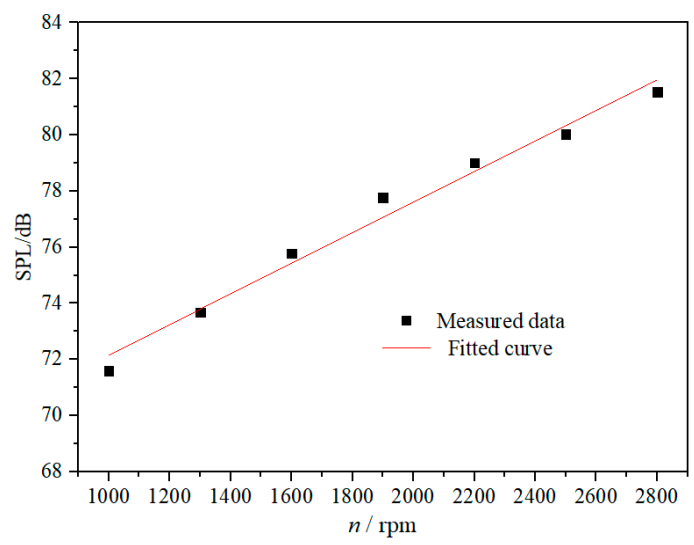

Figure 18. The radiated noise of the multi-stage centrifugal pump at different speeds. 


\subsection{Directivity of Radiated Noise at Different Flowrates}

To analyze the contribution of the different types of sound sources, the sound pressure level in the hemispherical surface around the source is measured. Microphones are mounted equally in the mid-span surface of the model pump and these five microphones are $1 \mathrm{~m}$ away from the surface of the second diffuser.

Considering the number of the microphones is limited, an interpolation method is used to get the directivity of the radiated noise at different flow rates, as shown in Figure 19. The experimental and simulated data of directivity agree well, considering the effect of background noise. It is obvious that the dipole is the main sound source of the multi-stage centrifugal pump at the different flowrates. Additionally, directivity at the $0.8 Q_{\mathrm{d}}$ is vertical, which explains that the directivity varies with the change of the flowrate.

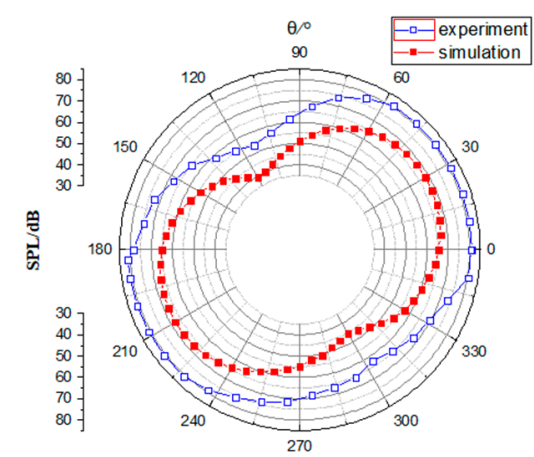

(a) $0.6 Q_{\mathrm{d}}$

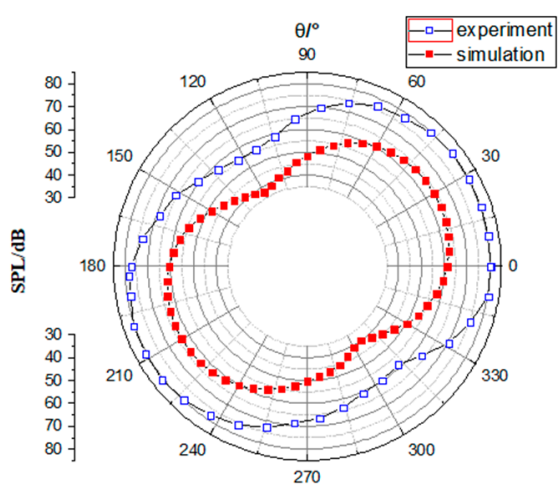

(c) $1.0 Q_{\mathrm{d}}$

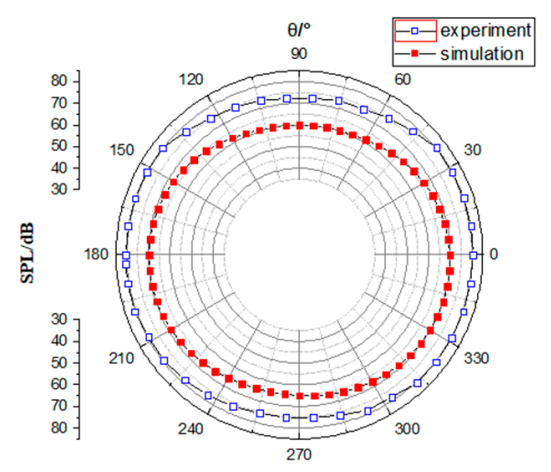

(b) $0.8 Q_{\mathrm{d}}$

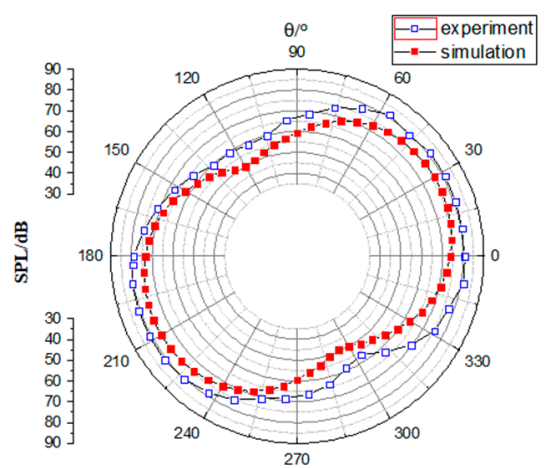

(d) $1.2 Q_{\mathrm{d}}$

Figure 19. Directivity of radiated noise at different flowrates.

\section{Hydraulic Optimization Design}

From the above experimental and numerical analysis, the main frequency of pressure pulsation and radiation noise of the prototype model pump is the blade passing frequency, and the amplitude under it is the largest. The flow passage is too narrow due to the unreasonable design of the number of blades of the impeller and the vane, which leads to poor fluid permeability. There are many vortices in the flow passage of the prototype pump model, and the intensity of its pressure pulsation is large, thereby generating a large radiated noise. 
In general, the main measures for reducing the radiated noise of the multi-stage pump include changing the rotational speed and process hydraulic optimization. Optimizing the effect of rotor-stator interaction is the most important region for the hydraulic design of a low noise multi-stage pump because the rotating speed is generally determined by the matching motor. Since the matching between the impeller and the guide vane of the multi-stage centrifugal pump also has a great influence on the pump performance, this study starts with the internal flow improvement and carries out a multi-objective design for the head, efficiency, and noise. The best match between the impeller and the guide vane is derived according to Formula (24) and (25). Finally, the number of impeller blades is six and the vane blade number is nine through mathematical calculation. Other geometric parameters were obtained by the velocity coefficient design method of the centrifugal pump and orthogonal optimization design [1].

$$
\begin{aligned}
H_{\text {impeller }} & =\frac{u_{2}}{g}\left(\sigma u_{2}-\frac{Q \cot \beta_{2}}{\pi D_{2} b_{2} \psi_{2}}\right) \\
H_{\text {vane }} & =\frac{\omega}{g} \frac{Q}{a_{3} b_{3} Z_{\text {vane }}} \frac{D_{3}}{2}
\end{aligned}
$$

The numerical calculation process including fluid calculation domain modeling, meshing, boundary condition setting, and flow field calculation is the same as before. Figure 20 gives the simulation results of the optimized pump. Seen from the calculated pump performance, the head and efficiency are higher than the original one after optimization. The efficiency after optimization increased by $12.9 \%$ under design flow. At the same time, the optimized multi-stage centrifugal pump achieves the highest efficiency around $1.1 Q_{\mathrm{d}}$, and the highest efficiency zone is widened.

The optimized multi-stage centrifugal pump pressure pulsation intensity shown in Figure 20c has the same characteristics as the original pump, that is, the pressure pulsation intensity in the impeller is the smallest, the pressure pulsation intensity in the positive guide vane is the largest, and the maximum value still appears in the positive guide vane passage near the throat at all stages. The pressure pulsation intensity of the optimized multi-stage centrifugal pump is reduced correspondingly to the same position as the original pump. Figure 20d revels that the dominant frequency of the radiated noise of the optimized multi-stage centrifugal pump is still blade passing frequency, but the sound pressure level under it is reduced by $2 \mathrm{~dB}$ compared with the original one shown in Figure 12c, which indicates that matching the relationship between the impeller and the guide vane is better after optimization. Reasonably, comparing the spectrum before and after optimization, it can be found that the magnitude of the radiated noise of the optimized multi-stage centrifugal pump in the low-frequency region is significantly reduced.

Finally, the optimized design was manufactured and sent to the product quality inspection center for a pump performance test. The multi-stage centrifugal pump after optimization has improved the head by $4.62 \mathrm{~m}$, the efficiency by $11.57 \%$, and reduced the average total sound pressure level by $2.6 \mathrm{~dB}$ at the design flow rate, which indicates that the simulation process and optimization method proposed in this paper is suitable for the pump designer. 


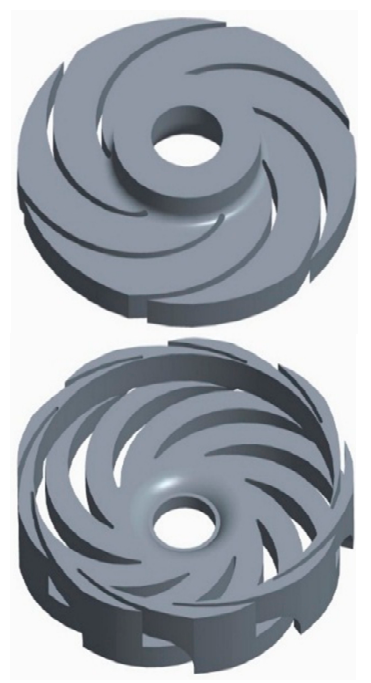

(a) flow domain impeller and guide vane

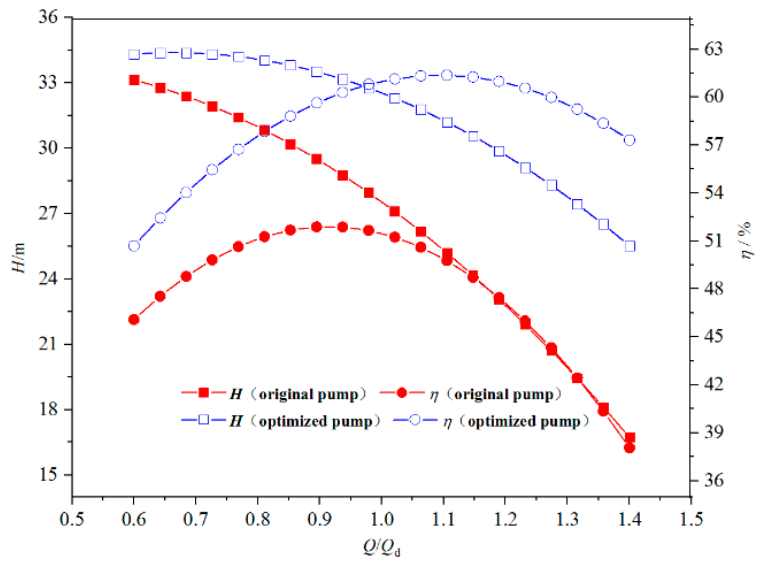

(b) pump performance

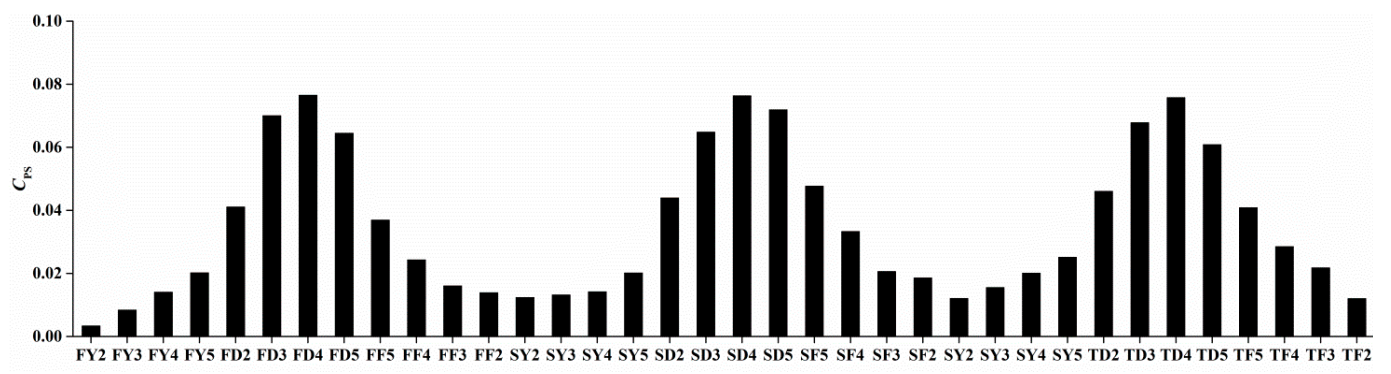

(c) amplitude distribution of optimized pump pressure fluctuation at $1.0 Q_{\mathrm{d}}$

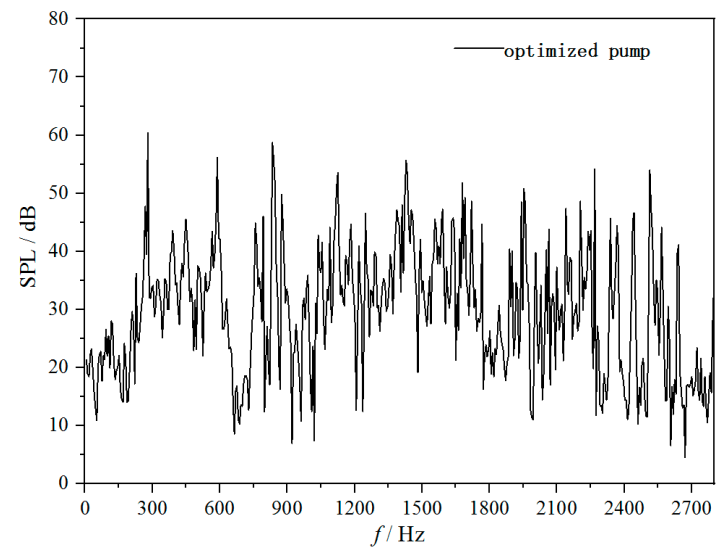

(d) frequency response curves of the optimized pump noise at $1.0 Q_{d}$

Figure 20. Simulation results of the optimized pump.

\section{Conclusions}

The study presents the acoustic simulation and experiment of radiated noise in a multi-stage pump. The following results were obtained:

(1) The sound pressure level in the multi-stage pump increases first and decreases afterward with the increment of the flowrate. The sound pressure level reaches its lowest value at $0.8 Q_{d}$, which corresponds to maximum efficiency working conditions. The sound pressure level of the radiated noise in the multistage pump rises linearly with the increase of the rotational speed. 
(2) The radiated noise of the multi-stage pump is characterized by dipoles. Furthermore, the main frequency of the radiated noise is the blade passing frequency $(327 \mathrm{~Hz})$. This fact proves that the rotor-stator interaction between impeller and diffuser is still the main hydraulic exciting force and sound source. In addition, the directivity of the sound source changes with the variation of the flowrate.

(3) Flow-induced radiated noise of the multi-stage centrifugal pump was calculated by the combined CFD(DES)/CA(FEM) method based on the Lighthill acoustic analogy theory. Comparisons of numerical predictions with the measured/analytical results reveal that the model can yield good results on the noise and the flow field. The most important aspect of the hydraulic design of a low-noise multi-stage centrifugal pump is to select the appropriate number of impeller blades and its matched guide vane.

Author Contributions: Q.S. and C.W. conceived and designed the experiments; G.L. performed the experiments and simulation; B.W. and K.H. analyzed the data; Q.S. wrote the paper; J.Y. funding acquisition.

Funding: This research was funded by National Key R\&D Program of China (2018YFC0810505), National Natural Science Foundation of China (51976079, 51779107), Senior Talent Foundation of Jiangsu University (15JDG048), Open Foundation of National Research Center of Pumps, Jiangsu University (NRCP201604).

Conflicts of Interest: The authors declare no conflict of interest.

\section{References}

1. Wang, C.; Shi, W.; Wang, X.; Jiang, X.; Yang, Y.; Li, W.; Zhou, L. Optimal design of multistage centrifugal pump based on the combined energy loss model and computational fluid dynamics. Appl. Energy 2017, 187, 10-26. [CrossRef]

2. Wang, C.; He, X.; Zhang, D.; Hu, B.; Shi, W. Numerical and experimental study of the self-priming process of a multistage self-priming centrifugal pump. Int. J. Energy Res. 2019, 43, 4074-4092. [CrossRef]

3. Huang, K.; Yuan, J.; Si, Q.; Lin, G. Numerical simulation on pressure pulsation in multistage centrifugal pump under several working conditions. J. Drain. Irrig. Mach. Eng. 2019, 37, 387-392.

4. Wang, C.; Hu, B.; Zhu, Y.; Wang, X.; Luo, C.; Cheng, L. Numerical study on the gas-water two-phase flow in the self-priming process of self-priming centrifugal pump. Processes 2019, 7, 330. [CrossRef]

5. Rochecarrier, N.L.; Ngoma, G.D.; Ghie, W. Numerical Investigation of a first stage of a multistage centrifugal pump: Impeller, diffuser with return vanes, and casing. Isrn Mech. Eng. 2015, 2013, 578072.

6. Wang, C.; He, X.; Shi, W.; Wang, X.; Wang, X.; Qiu, N. Numerical study on pressure fluctuation of a multistage centrifugal pump based on whole flow field. Aip Adv. 2019, 9, 035118. [CrossRef]

7. Zhang, J.; Xia, S.; Ye, S.; Xu, B.; Song, W.; Zhu, S.; Xiang, J. Experimental investigation on the noise reduction of an axial piston pump using free-layer damping material treatment. Appl. Acoust. 2018, 139, 1-7. [CrossRef]

8. Ye, S.; Zhang, J.; Xu, B.; Zhu, S. Theoretical investigation of the contributions of the excitation forces to the vibration of an axial piston pump. Mech. Syst. Signal Process. 2019, 129, 201-217. [CrossRef]

9. Wang, J.; Feng, T.; Liu, K.; Zhou, Q.-J. Experimental Research on the Relationship between the Flow-induced Noise and the Hyaraulic Parameters in Centrifugal Pump. Fluid Mach. 2007, 6, 1-13.

10. Brennen, C.E. A review of the dynamics of cavitating pumps. J. Fluids Eng. 2013, 135, 061301. [CrossRef]

11. He, X.; Jiao, W.; Wang, C.; Cao, W. Influence of surface roughness on the pump performance based on Computational Fluid Dynamics. IEEE Access 2019, 7, 105331-105341. [CrossRef]

12. Qian, J.Y.; Chen, M.R.; Liu, X.L.; Jin, Z.J. A numerical investigation of the flow of nanofluids through a micro Tesla valve. J. Zhejiang Univ. Sci. A 2019, 20, 50-60. [CrossRef]

13. Qian, J.Y.; Gao, Z.X.; Liu, B.Z.; Jin, Z.J. Parametric study on fluid dynamics of pilot-control angle globe valve. ASME J. Fluids Eng. 2018, 140, 111103. [CrossRef]

14. Zhu, Y.; Tang, S.; Wang, C.; Jiang, W.; Yuan, X.; Lei, Y. Bifurcation Characteristic research on the load vertical vibration of a hydraulic automatic gauge control system. Processes 2019, 7, 718. [CrossRef]

15. Zhu, Y.; Tang, S.; Quan, L.; Jiang, W.; Zhou, L. Extraction method for signal effective component based on extreme-point symmetric mode decomposition and Kullback-Leibler divergence. J. Braz. Soc. Mech. Sci. Eng. 2019, 41, 100. [CrossRef] 
16. Zhu, Y.; Qian, P.; Tang, S.; Jiang, W.; Li, W.; Zhao, J. Amplitude-frequency characteristics analysis for vertical vibration of hydraulic AGC system under nonlinear action. Aip Adv. 2019, 9, 035019. [CrossRef]

17. Chu, S.; Dong, R.; Katz, J. Relationship Between Unsteady Flow, Pressure Fluctuations, and Noise in a Centrifugal Pump—Part A: Use of PDV Data to Compute the Pressure Field. J. Fluids Eng. 1995, 117, $24-29$. [CrossRef]

18. Chu, S.; Dong, R.; Katz, J. Relationship between Unsteady Flow, Pressure Fluctuations, and Noise in a Centrifugal Pump-Part B: Effects of Blade-Tongue Interactions. J. Fluids Eng. 1995, 117, 30-35. [CrossRef]

19. Khalifa, A.E.; Al-Qutub, A.M.; Ben-Mansour, R. Study of Pressure Fluctuations and Induced Vibration at Blade-Passing Frequencies of a Double Volute Pump. Arab. J. Sci. Eng. 2011, 36, 1333-1345. [CrossRef]

20. Parrondo, J.; Pérez, J.; Barrio, R.; Gonzales, J. A simple acoustic model to characterize the internal low frequency sound field in centrifugal pumps. Appl. Acoust. 2011, 72, 59-64. [CrossRef]

21. Liu, H.L.; Dai, H.W.; Ding, J. Numerical and experimental studies of hydraulic noise induced by surface dipole sources in a centrifugal pump. J. Hydrodyn. Ser. B 2016, 28, 43-51. [CrossRef]

22. Howe, M.S. On the Estimation of Sound Produced by Complex Fluid-Structure Interactions, with Application to a Vortex Interacting with a Shrouded Rotor. Proc. Math. Phys. Sci. 1991, 433, 573-598. [CrossRef]

23. Kato, C.; Yoshimura, S.; Yamade, Y.; Jiang, Y.Y.; Wang, H.; Imai, R.; Katsura, H.; Yoshida, T.; Takano, Y. Prediction of the Noise from a Multi-Stage Centrifugal Pump. In Proceedings of the ASME 2005 Fluids Engineering Division Summer Meeting, Houston, TX, USA, 19-23 June 2005; pp. 1273-1280.

24. Ding, J.; Liu, H.L.; Wang, Y.; Tan, M.G.; Cui, J.B. Numerical study on the effect of blade outlet angle on centrifugal pump noise. J. Vib. Shock 2014, 33, 122-127.

25. Si, Q.R.; Ali, A.; Yuan, J.P.; Fall, I.; Muhammad, Y.F. Flow-induced noises in a centrifugal pump: A review. Sci. Adv. Mater. 2019, 11, 909-924. [CrossRef]

26. Keller, J.; Barrio, R.; Parrondo, J.; Barrio, R.; Fernandez, J.; Blanco, E. Effects of the Pump-Circuit Acoustic Coupling on the Blade-Passing Frequency Perturbations. Appl. Acoust. 2014, 76, 150-156. [CrossRef]

27. Gao, M.; Dong, P.; Lei, S.; Turan, A. Computational Study of the Noise Radiation in a Centrifugal Pump When Flow Rate Changes. Energies 2017, 10, 221. [CrossRef]

28. Jiang, Y.Y.; Yoshimura, S.; Imai, R.; Katsura, H.; Yoshida, T.; Kato, C. Quantitative evaluation of flow-induced structural vibration and noise in turbomachinery by full-scale weakly coupled simulation. J. Fluids Struct. 2007, 23, 531-544. [CrossRef]

29. Timushev, S. Development and Experimental Validation of 3D Acoustic-Vortex Numerical Procedure for Centrifugal Pump Noise Prediction. In Proceedings of the ASME 2009 Fluids Engineering Division Summer Meeting, Vail, CO, USA, 2-6 August 2009; pp. 389-398.

30. Barrio, R.; Parrondo, J.; Blanco, E. Numerical analysis of the unsteady flow in the near-tongue region in a volute-type centrifugal pump for different operating points. Comput. Fluids 2010, 39, 859-870. [CrossRef]

31. Dong, L.; Dai, C.; Kong, F.; Fu, L.; Bai, Y. Impact of blade outlet angle on acoustic of centrifugal pump as turbine. Trans. Chin. Soc. Agric. Eng. 2015, 31, 69-75.

32. Rui, X.P.; Zhao, Y. Numerical simulation and experimental research of flow-induced noise for centrifugal pumps. J. Vibroeng. 2016, 18, 622-636.

33. Hao, Z. Vibration Noises and Acoustic Optimization Design of the Centrifugal Pump. In Proceedings of the International Conference on Machinery, Materials and Computing Technology, Hangzhou, China, 23-24 January 2016.

34. Heng, Y.; Yuan, S.; Hong, F.; Yuan, J.; Si, Q.; Hu, B. A Hybrid Method for Flow-Induced Noise in Centrifugal Pumps Based on LES and FEM. In Proceedings of the ASME 2013 Fluids Engineering Division Summer Meeting, Vail, CO, USA, 2-6 August 2013; p. V01BT10A034.

35. Tu, S.; Shahrouz, A.; Reena, P.; Watts, M. An implementation of the Spalart-Allmaras DES model in an implicit unstructured hybrid finite volume/element solver for incompressible turbulent flow. Int. J. Numer. Methods Fluids 2010, 59, 1051-1062. [CrossRef]

36. Gülish, J.F. Centrifugal Pumps; Springer: Berlin/Heidelberg, Germany; New York, NY, USA, 2008.

37. Methods of Measuring and Evaluating Noise of Pumps; China Standard Press: Beijing, China, 2013; GB/T $29529-2013$. 